\title{
Análisis comparativo de la estructura, diversidad y composición de comunidades arbóreas de un paisaje agropecuario en Tabasco, México
}

\section{Comparative analysis of structure, diversity and composition of tree communities in an agricultural landscape of Tabasco, Mexico}

\author{
Noel González-Valdivia ${ }^{1}$, Susana Ochoa-Gaona ${ }^{1}$, Bruce G. Ferguson ${ }^{2}$, Carmen Pozo $^{3}$, Christian \\ Kampichler ${ }^{4}$ e Isidro Pérez-Hernández ${ }^{1}$ \\ ${ }^{1}$ El Colegio de la Frontera Sur, Sistemas de Producción Alternativos. Apartado postal 1042, Administración de Correos 2000, 86031 Villahermosa, \\ Tabasco, México. \\ ${ }^{2}$ El Colegio de la Frontera Sur, Sistemas de Producción Alternativos. Carretera Panamericana y Periférico Sur s/n, María Auxiliadora, 29290 San \\ Cristóbal de las Casas, Chiapas. \\ ${ }^{3}$ El Colegio de la Frontera Sur, Conservación de la Biodiversidad. Av. del Centenario Km. 5.5, 77900 Chetumal, Quintana Roo, México. \\ ${ }^{4}$ División de Ciencias Biológicas, Universidad Juárez Autónoma de Tabasco. Carretera Villahermosa-Cárdenas Km. $0.5 \mathrm{~s} / \mathrm{n}$, entronque a Bosques de \\ Saloya, 86150 Villahermosa, Tabasco, México. \\ 凶sochoa@ecosur.mx
}

\begin{abstract}
Resumen. Con el objetivo de evaluar el efecto del manejo sobre la diversidad, estructura y composición de la flora arbórea nativa en un paisaje montañoso neotropical, se estudiaron 2 ecomosaicos: selva mediana y su matriz agropecuaria. Se inventariaron 96 parcelas registrando 172 especies. Los ecomosaicos difieren en riqueza, abundancia de especies y estructura como resultado del manejo del paisaje. La composición de los remanentes de selva se muestra como extrema de los potreros. En la vegetación secundaria madura se encontró la mayor riqueza (92 especies), mientras que los potreros fueron más pobres en especies. La flora arbórea de selva está representada en un $66 \%$ dentro de la matriz, particularmente en la vegetación secundaria; 27 especies son características de las unidades de paisaje, la mayor parte son zoocoras y están asociadas a comunidades maduras. Se encontró que hay complementariedad entre los ecomosaicos, la cual está relacionada con el hecho de que los campesinos mantienen áreas de reserva de selva, tanto en un núcleo compacto como en fragmentos distribuidos a lo largo del paisaje. Sin embargo, las tendencias indican que el sistema de acahuales tiende a desaparecer, lo que homogeneizará la matriz y aumentará el contraste entre ecomosaicos, comprometiendo la regeneración de la selva y de las especies asociadas al mismo.
\end{abstract}

Palabras clave: biodiversidad, bosque tropical, flora leñosa, neotrópico, paisaje cultural, vegetación secundaria.

\begin{abstract}
In order to evaluate the effect of management on diversity, structure and composition of native arboreal flora in a mountainous Neotropical landscape, we studied 2 ecomosaics: montane rain forest, and the surrounding agricultural matrix. We inventoried 96 plots, registering 172 species. As a result of management, the ecomosaics differed in species richness and abundance as well as in vegetation structure, with forest remnants and pastures as the 2 extremes. Mature secondary vegetation had the most tree species (92), and pastures the fewest. Two thirds of forest tree species were also represented in the surrounding matrix, especially in fallows. Twenty-seven species were characteristic of particular landscape units. Most of these were zoochores associated with mature forest. The actual landscape management maintains a certain proportion of conserved habitats and dispersed forest remnants which enrich the agricultural matrix. However the tendency toward less fallow area and more pasture indicates a transition toward a more homogeneous agricultural matrix, less forest regrowth and greater contrast between forest and agricultural ecomosaics. This will increase the contrast between the ecomosaics and threaten the regeneration of mature forest and its associated species
\end{abstract}

Key words: biodiversity, cultural landscape, Neotropics, secondary vegetation, tropical forest, woody flora.

\section{Introducción}

La actividad agropecuaria en el trópico ha transformado los paisajes boscosos simplificando su

Recibido: 21 enero 2009; aceptado: 17 febrero 2010 biodiversidad (Isaac-Márquez et al., 2005; Sánchez et al., 2005). En el sureste mexicano, y particularmente en el estado de Tabasco, las selvas han sido transformadas a pasturas, las que actualmente cubren el $95 \%$ de la extensión estatal (Hernández-Daumás, 2005) y han reducido hasta casi su desaparición el bosque tropical (SEDESPA, 2006). 
Diversos estudios sobre la flora y fauna remanente en los sistemas agropecuarios han evidenciado la capacidad de la biota autóctona para utilizar ambientes modificados por el hombre y mantenerse a través del tiempo (Vílchez et al., 2004; Sekercioglu et al., 2007; Tobar et al., 2007; Vandemeer y Perfecto, 2007), manifestando indicios de resiliencia. Dicha capacidad es relevante para la ecología del paisaje y la restauración ecológica, pues abre la posibilidad de diseñar la agricultura, ganadería y forestería tropical de manera que cumplan con los objetivos socioeconómicos y ecológicos en el marco del manejo sustentable de los recursos (Harvey et al., 2008).

El estudio de patrones de paisaje permite profundizar en el conocimiento y técnicas de manejo sustentable de los recursos naturales a diferentes escalas (Ortiz-Espejel y Toledo, 1998; Masera et al., 1999), especialmente cuando se encuentran modelos de paisaje complejos generados por la cultura local (Stevens, 1996; Toledo, 2002). Identificar y estudiar estos modelos es una necesidad urgente en el trópico. Es igualmente relevante documentar los remanentes de bosque como referentes ecológicos e históricos para tener una base de comparación en la evaluación de las comunidades forestales tropicales con respecto a sus agrosistemas (Higgs, 2003; Martínez, 2003). Este tipo de investigación incrementa sus alcances si además de estudiar el efecto que los paisajes y sus elementos tienen sobre especies particulares, se evalúa cómo influyen éstos sobre las comunidades -así lo sugieren Gutiérrez et al. (2001)- lo que permite mejorar la base científica con que se formulan las recomendaciones que orientan la política ambiental en el trópico americano. El objetivo de la investigación fue evaluar si el manejo actual de un paisaje montañoso neotropical permite la conservación de la diversidad, estructura y composición de la flora arbórea nativa, propia de la selva mediana perennifolia.

\section{Materiales y métodos}

Descripción de la zona de estudio. El ejido Niños Héroes de Chapultepec (en adelante NHC), municipio de Tenosique, Tabasco, se localiza al SE de México se ubica en los $17^{\circ} 15^{\prime} 00^{\prime \prime}-17^{\circ} 40^{\prime} 48^{\prime \prime} \mathrm{N}$ y $90^{\circ} 59^{\prime} 09^{\prime \prime}-91^{\circ} 38^{\prime} 16^{\prime \prime} \mathrm{O}$, con una superficie territorial de 2066 ha (Fig. 1; OchoaGaona et al., 2008). Fue fundado en la década de 1970 por colonos migrantes de México y de Guatemala (Hernández-Daumás, 2005). Se encuentra dentro del pequeño macizo montañoso con altura máxima de $700 \mathrm{~m}$, sobre rocas cársticas, suelos delgados y con un clima cálido y húmedo de $26 \mathrm{C}^{\circ}$ de temperatura promedio y precipitación media anual de $2750 \mathrm{~mm}$ (Isaac-Márquez et al., 2005). Las principales actividades son pecuarias y de manejo forestal, con agricultura de subsistencia en menor escala (Isaac-Márquez et al., 2008), que mantienen una reserva ecológica ejidal ocupada por selva mediana perennifolia, la cual es biológica y estructuralmente muy diversa (Vázquez-Negrin y Castillo-Acosta, 2007; Ochoa-Gaona et al., 2008). La zona forma parte del Área Natural Protegida Cañón del Usumacinta (SEMARNAT, 2008) que está conectada con El Petén guatemalteco y la Selva Lacandona.

Los remanentes de bosque tropical se encuentran inmersos en un mosaico de vegetación secundaria de diferente edad derivada de la actividad agrícola y pastizales inducidos para la ganadería localizados en las partes planas u onduladas (Isaac-Márquez et al., 2005). Estos remanentes de bosque tropical perennifolio alcanzan en su mejor estado 30 metros de altura y están conformados por 3 estratos arbóreos y 1 herbáceo compuesto por aráceas, marantáceas y helechos; además, se presentan lianas y varios tipos de orquídeas (Gobierno del Estado de Tabasco, 1997). De las especies arbóreas más comunes se pueden mencionar Swietenia macrophylla (caoba), Cedrela odorata (cedro), Andira galeottiana (macayo), Spondias mombin (jobo), Tabebuia rosea (macuilís), Ceiba pentandra (ceiba), Nectandra ambigens (laurel), Castilla elastica (árbol de hule), Calophyllum brasiliense (barí) y Cordia alliodora (bojón; Ochoa-Gaona et al., 2008).

Diseño de muestreo. La composición y la estructura arbórea en el paisaje fueron evaluadas a partir de la información obtenida de 8 unidades de paisaje (sensu Zonneveld, 1989); 4 se anidaron en el ecomosaico de la matriz agropecuaria (MA) y 4 en el ecomosaico de selva mediana perennifolia, como referencia ecológica (RE; sensu Higgs, 2003); éstas se localizaron en el terreno, con base en la sugerencia de los ejidatarios.

Las unidades se presentan en una secuencia de acuerdo con su posición respecto al relieve del terreno. Ocupan en la MA desde las partes de mayor altitud hacia las más bajas: acahual maduro (AM), acahual joven (AJ), potrero con árboles dispersos (PAD) y árboles en línea (AL), ocupando los 2 últimos las partes planas. Para los remanentes de selva, las unidades en ese mismo orden posicional fueron: selva en las cimas, como referente del acahual maduro ( $\left.\mathrm{RE}_{\mathrm{AM}}\right)$; selva en laderas, como referente del acahual joven $\left(\mathrm{RE}_{\mathrm{AJ}}\right)$; selva en pie de monte o planicie, como referente de los potreros con árboles $\left(\mathrm{RE}_{\mathrm{PAD}}\right)$, y elementos de perturbación lineal, como referente de los árboles en línea (REL) o senderos de acceso dentro de la selva (Cuadro 1).

Se establecieron 3 parcelas rectangulares de $100 \mathrm{~m}$ x $5 \mathrm{~m}$ (Ochoa-Gaona et al., 2004) dentro de cada unidad del paisaje, separadas al menos por $50 \mathrm{~m}$ entre sí. De cada unidad de paisaje se hicieron 4 réplicas, separadas 


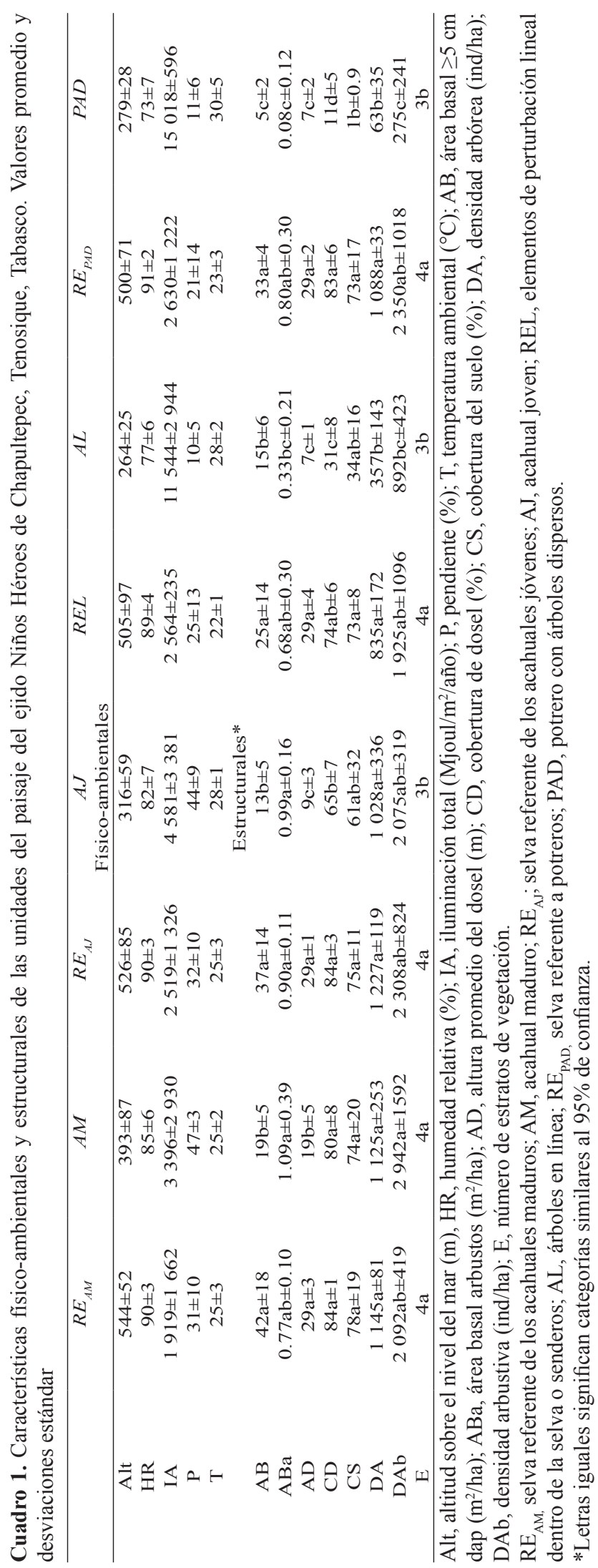

por una distancia mínima de $300 \mathrm{~m}$ entre sí; en total se muestrearon 96 parcelas. Se midió el diámetro a la altura del pecho (dap) de los individuos con dap $\geq 5 \mathrm{~cm}$. Los juveniles incluyeron los de dap $<5 \mathrm{~cm} \mathrm{y} \geq 1.5 \mathrm{~m}$ de altura, los que se contaron en subparcelas de $20 \mathrm{~m} \times 5 \mathrm{~m}$ en el centro de las parcelas. Las plántulas $(<1.5 \mathrm{~m}$ de altura) se registraron en subparcelas de $2 \mathrm{~m} \times 2 \mathrm{~m}$ localizadas en cada extremo de la parcela. El análisis de estructura se basó en Godínez-Ibarra y López-Mata (2002), EscobarOcampo y Ochoa-Gaona (2007) y Zamora-Crescencio et al. (2008).

Con base en el marco lógico desarrollado por Smith et al. (1998), se formaron los siguientes grupos funcionales de tipo de árbol: árbol emergente $(>35 \mathrm{~m})$, árbol persistente alto $(\geq 20 \mathrm{~m})$, árbol persistente bajo $(<20 \mathrm{~m})$, árbol pionero, arbusto alto (entre 1.5 y $5 \mathrm{~m}$ ), arbusto bajo $(<1.5 \mathrm{~m})$, arbusto pionero, palma, y árbol introducido (cultivado).

La determinación de los síndromes y mecanismos de dispersión de semillas o frutos se basó principalmente en Alcázar (2007) enriquecido con elementos tomados de Snow (1981), Budke et al. (2005), Kinoshita et al. (2006) y Silva et al. (2009). Se identificaron los siguientes mecanismos de dispersión: sólo por vertebrados no voladores (principalmente mamíferos), sólo por vertebrados voladores (principalmente aves), tanto por vertebrados no voladores como voladores, por viento (anemocoria), autocoria por gravedad, autocoria explosiva, y sólo dispersada por humanos (antropocoria).

Análisis de datos. Los datos se analizaron con base en los árboles con dap $\geq 5 \mathrm{~cm}$, excepto cuando se aclara. La eficiencia del muestreo se evaluó por medio de curvas de acumulación de especies utilizando EstimateS 8.0 (Colwell, 2006) y aplicando la ecuación propuesta por Clench (1978). Las variables estructurales: área basal, altura promedio del dosel, cobertura del dosel, cobertura del suelo, densidad y número de estratos fueron evaluadas mediante análisis de varianza (Andeva, $\alpha=0.05$ ) y sus medias agrupadas por Tukey, previa determinación de normalidad mediante la prueba de Kolmogorov-Smirnov y de homocedasticidad con la prueba Levene (Habit et al., 2003) utilizando SPSS 10.0 (Morrison, 1999).

La similitud entre hábitats fue comparada mediante análisis de agrupamiento UPGMA (unweighted pair-group average), utilizando como medida de distancia el índice de Bray-Curtis - que considera el valor cuantitativo del dato y es asimétrico por no incluir los pares de ausencias en la similitud (Zuur et al., 2007) y los índices de diversidad de Shannon-Wiener base logaritmo natural, dominancia de Simpson y equitatividad de Pielou, para comparar las comunidades bióticas (Magurran, 1988; Spellerberg, 2005; Pérez et al., 2007), 

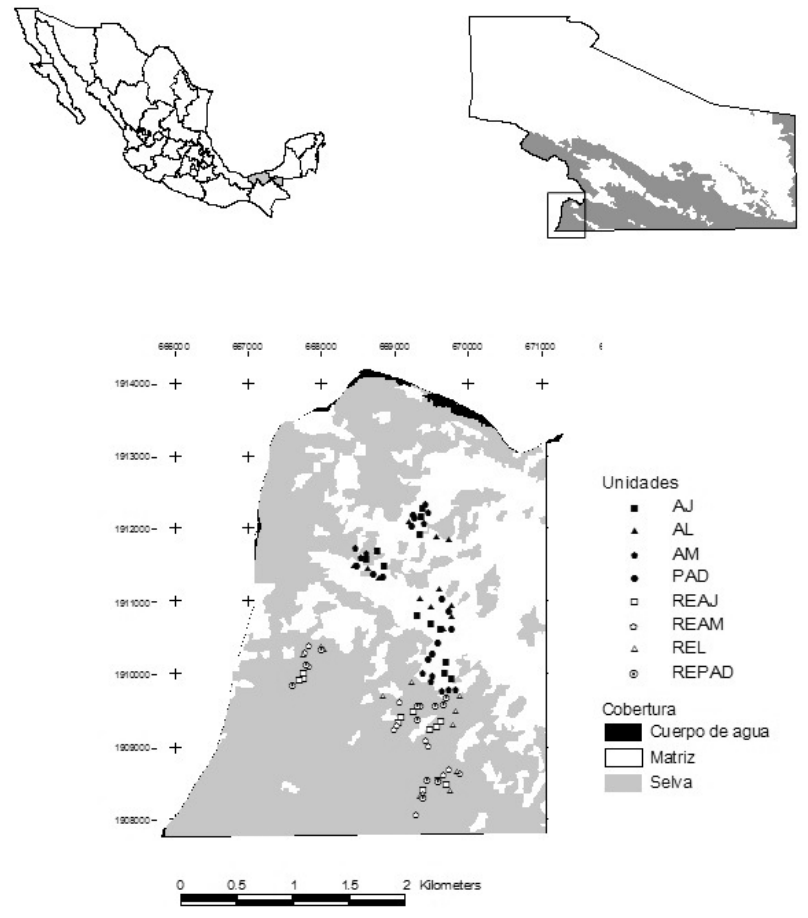

Figura 1. Localización del área de estudio. A, Tabasco, México; $\mathrm{B}$, municipio de Tenosique, marcada el área de Reserva del Cañón del Usumacinta; en el recuadro, ubicación del ejido Niños Héroes de Chapultepec; C, puntos de conteo establecidos en la selva y la matriz agropecuaria.

se calcularon mediante el programa PAST 2.0 (Hammer et al., 2001).

Los datos de abundancia se exploraron mediante la prueba Box y Cox (1964) y se transformaron a raíz cuadrada siguiendo la ley de Taylor (Herrando-Pérez, 2002). El análisis de correspondencia canónica (ACC) se realizó mediante Canoco 4.5 (Lepš y Šmilauer, 2005), buscando relaciones entre los grupos funcionales (dispersión y tipo de árbol) respecto a las variables ambientales y estructurales de cada unidad. Para determinar qué especies aportan más a la diferenciación entre unidades y ecomosaicos se analizaron similitud y disimilitud porcentual (SIMPER) con Primer 5.2.8 (Clarke y Gorley, 2006); el corte se hizo al obtener una contribución acumulativa $\geq 50 \%$. Se ingresaron las especies de mayor peso porcentual por unidad, y se buscó si éstas también eran características de otra unidad, aunque su contribución fuera baja.

\section{Resultados}

Eficiencia de muestreo. El muestreo de la vegetación alcanzó el 87\% de eficiencia al registrar 172 especies de las 197 esperadas en el paisaje del ejido. Se alcanzó el $77 \%$ de eficiencia en la matriz (128 especies registradas de las 167 esperadas). El muestreo en la selva mediana perennifolia (RE) logra capturar el $88 \%$ de las 141 especies de árboles esperadas, registrando 124 especies (Fig. 2).

Estructura. Todas las variables estructurales presentaron diferencias estadísticas significativas entre unidades de paisaje. El área basal arbórea $(\mathrm{F}=2.94, p=0.022)$, el área basal arbustiva $(\mathrm{F}=8.6, p<0.001)$, la densidad arbórea $(\mathrm{F}=22.96, p<0.000)$, la densidad de arbustos $(\mathrm{F}=4.9$, $p<0.001)$, la altura promedio del dosel $(\mathrm{F}=53.67, p$ $<0.000)$, el número de estratos $(\mathrm{F}=14, p<0.001)$ y la cobertura del dosel de la flora leñosa arbórea $(\mathrm{F}=87$, $p<0.001)$.

La prueba de Tukey muestra que los promedios de las variables estructurales tienen mayor similitud entre las unidades de selva y entre las unidades de vegetación secundaria (AM y $\mathrm{AJ}$ ) y que los pastizales con árboles dispersos mostraron diferencias significativas al resto de las unidades. El área basal del arbolado y la altura del dosel fueron diferentes entre las unidades de la selva respecto a las correspondientes unidades de la matriz. El área basal de arbustos, la densidad de árboles y la densidad de arbustos fue similar entre las unidades de selva y las unidades de vegetación secundaria (AJ y AM); mientras que los pastizales y los árboles en línea mostraron diferencias significativas respecto a su referencia ecológica. La cobertura del dosel y el número de estratos sólo fue similar entre el acahual maduro y su referente ecológico, siendo diferente entre el resto de unidades de la matriz con relación a su referente de selva. La cobertura del suelo, sólo mostró diferencias entre el pastizal y la selva referente (Cuadro 1).

Diversidad de especies. En las unidades del paisaje, sólo el acahual maduro es similar a su referente respecto al número de especies; el resto de pares de unidades matrizselva son diferentes. En cuanto al número de individuos, las unidades de vegetación secundaria se muestran similares a las de la selva. El índice de diversidad de Shannon-Wiener osciló entre las unidades de paisaje desde 2.66 en potreros hasta 3.84 para la selva referente de los acahuales jóvenes, mientras que el índice de dominancia se mantuvo por debajo de 0.09 , coherente con el índice de equitatividad, que fue mayor a 0.75 . Las unidades de acahual arbóreo joven y maduro contienen un número similar de especies al encontrado en las unidades de selva (Cuadro 2).

Composición de especies. De las 172 especies arbóreas, 82 se comparten en los 2 ecomosaicos, mientras que 42 sólo fueron registradas en la selva y 48 en la matriz agropecuaria (Apéndice 1). En los ecomosaicos, la selva y la matriz agropecuaria contienen 72 y $76 \%$ de la riqueza medida respectivamente. Mediante el análisis de disi- 
Cuadro 2. Índices de diversidad en unidades del paisaje del ejido Niños Héroes de Chapultepec, Tenosique, Tabasco

\begin{tabular}{lcccccccc}
\hline Parámetro/Unidad & $R E_{A M}$ & $A M$ & $R E_{A J}$ & $A J$ & $R E L$ & $A L$ & $R E_{P A D}$ & $P A D$ \\
\hline Núm. de especies $^{1}$ & $88 \mathrm{a}$ & $92 \mathrm{a}$ & $86 \mathrm{a}$ & $74 \mathrm{~b}$ & $83 \mathrm{a}$ & $57 \mathrm{c}$ & $83 \mathrm{a}$ & $18 \mathrm{~d}$ \\
Núm. de individuos $^{2}$ & $687 \mathrm{a}$ & $675 \mathrm{a}$ & $736 \mathrm{a}$ & $617 \mathrm{a}$ & $501 \mathrm{a}$ & $214 \mathrm{~b}$ & $653 \mathrm{a}$ & $38 \mathrm{~b}$ \\
Diversidad de Shannon & 3.74 & 3.68 & 3.84 & 3.21 & 3.58 & 3.43 & 3.73 & 2.66 \\
Dominancia de Simpson $^{\text {Equidad de Pielou }}$ & 0.04 & 0.05 & 0.03 & 0.09 & 0.05 & 0.06 & 0.04 & 0.09 \\
\hline
\end{tabular}

${ }^{1}$ Letras diferentes indican categorías diferentes $(\mathrm{F}=22, \mathrm{p}<.001)$

${ }^{2}$ Letras diferentes indican categorías diferentes $(\mathrm{F}=23, \mathrm{p}<.001)$; en 0.6 ha de muestreo por unidad.

$\mathrm{AM}$, acahual maduro; AJ, acahual joven; $\mathrm{AL}$, árboles en línea; $\mathrm{PAD}$, potrero con árboles dispersos; $\mathrm{RE}_{\mathrm{AM}}$, selva referente de los acahuales maduros; $\mathrm{RE}_{\mathrm{AJ}}$, selva referente de los acahuales jóvenes; $\mathrm{RE}_{\mathrm{PAD}}$, selva referente a potreros; $\mathrm{REL}$, elementos de perturbación lineal dentro de la selva o senderos.
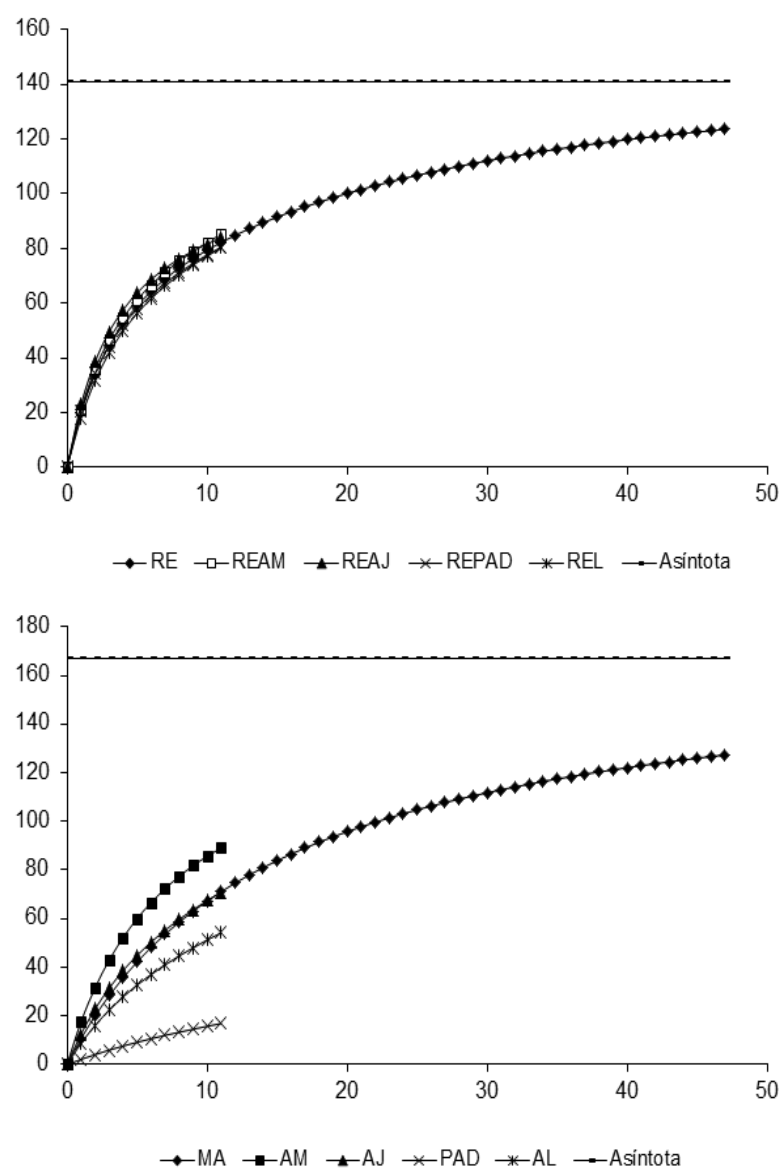

Figura 2. Acumulación de especies arbóreas en la matriz agropecuaria y en los remanentes de selva, así como las 4 unidades anidadas en cada uno de estos ambientes en el paisaje en función del número de parcelas de muestreo (eje X). A: RE, selva mediana perennifolia o referente ecológico; REAM, selva referente de los acahuales maduros; REAJ, selva referente de los acahuales jóvenes; REPAD, selva referente a potreros; REL, elementos de perturbación lineal dentro de la selva o senderos. B: MA, matriz agropecuaria; AJ, acahual joven; AL, árboles en línea; AM, acahual maduro; PAD, potreros con árboles dispersos. militudes porcentuales se determinó que las unidades de la matriz agropecuaria y sus contrapartes en la selva resultaban diferentes al compararse de manera pareada. Las diferencias entre unidades AM-REAM, AJ-REAJ, PAD-REPAD y AL-REL alcanzaron 81.2, 95.5, 99.4 y $92.8 \%$ de disimilitud, respectivamente $(\mathrm{R}$ global $=0.62$, sig.=0.03). El análisis de agrupamiento de las unidades mediante el índice de Bray-Curtis, separa 4 grupos distintos. Uno está conformado por las unidades de la selva (índice de similitud mayor de 0.70), exceptuando los senderos en la selva (REL), con el que comparten el 55\% de su ensamble. Otro grupo lo conforman los acahuales maduros y jóvenes, los que comparten una similitud del $40 \%$ con la selva. Los árboles en línea y los potreros con árboles dispersos, se mantienen como grupos separados. La vegetación arbustiva y juvenil $(1.5 \mathrm{~cm} \leq \mathrm{DAP}<5 \mathrm{~cm})$ repite el patrón de agrupamiento (Fig. 3).

Respuesta de grupos funcionales. Por su forma de dispersión, las especies arbóreas del ejido son zoocoras (128 spp., 74\%), anemocoras (29 spp., 17\%), autocoras (12 spp., 7\%), cultivadas (3 spp., 2\%). En la selva 99 especies fueron zoocoras, 17 anemocoras y 7 autocoras. En la matriz agropecuaria 94 especies fueron zoocoras, 23 anemocoras, 11 autocoras y 3 dispersadas por el hombre (Apéndice 1). Estos grupos funcionales mostraron diferencias $(\mathrm{F}=15.06, p=0.002)$; el primer eje explicó el $47 \%$ y el segundo el $20 \%$ de la variación mostrada por las especies; en el mismo orden, se explica el $64 \%$ y el $27 \%$ de la relación entre el grupo de dispersión con su ambiente (Fig. 4).

Por tipo de árbol, los grupos funcionales también mostraron diferencias $(\mathrm{F}=12.25, p=0.002)$. El primer eje explicó el $42 \%$ y el segundo el $8 \%$ de la variación mostrada por las especies y en el mismo orden se explica el $64 \%$ y el 13\% de la relación entre el tipo de árbol con su ambiente (Fig. 5). Los remanentes de selva y la vegetación secundaria concentran un mayor número de especies del tipo árbol persistente, tanto de los estratos altos como bajos, además de incluir el componente de palmas y lia- 


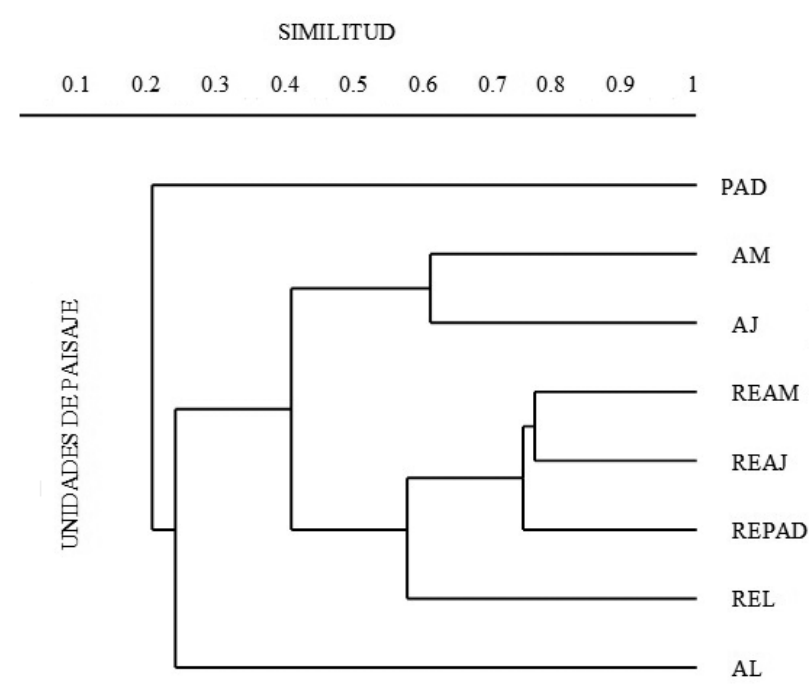

Figura 3. Agrupamiento de unidades en función de la similitud Bray-Curtis entre comunidades de árboles DAP $\geq 5$ centímetros. AM, acahual maduro; AJ, acahual joven; AL, árboles en línea; PAD, potrero con árboles dispersos; REAM, selva referente de los acahuales maduros; REAJ, selva referente de los acahuales jóvenes; REPAD, selva referente a potreros; REL, elementos de perturbación lineal dentro de la selva o senderos.

nas (DAP $\geq 5 \mathrm{~cm}$ ). En la matriz las especies pioneras, tanto de árboles como arbustos fueron predominantes (Apéndice 1).

Especies caracteristicas. Por otro lado, el análisis de similitud porcentual permitió distinguir 27 especies que son características de las unidades de paisaje; 4 caracterizan los potreros, 8 los árboles en línea, 10 los acahuales jóvenes, 10 los acahuales maduros, 11 los senderos en selva, 11 la selva referente de acahuales maduros, 13 la selva referente de acahuales jóvenes y 10 la selva referente de potreros. Sólo Swietenia macrophylla (caoba) y Cedrela odorata (cedro) se registraron como características de 1 unidad (AL), mientras que el resto se compartieron en 2, 3, 4 y hasta 5 unidades del paisaje. Al analizar por pares, REPAD-PAD no comparten ninguna especie, REL-AL y REAJ-AJ comparten 1 especie, REAM-AM comparten 2 especies. La mayor parte de los referentes de la selva comparten al menos 8 especies (Cuadro 3).

\section{Discusión}

Eficiencia de muestreo. El muestreo realizado en este trabajo es aceptable, puesto que según la comparación entre el ensamble identificado respecto al esperado se alcanzó hasta el $87 \%$ de la flora arbórea. Con base en las curvas de acumulación, se puede evaluar que las parcelas de selva en 0.1 ha contienen $\geq 42$ especies, un número similar al que

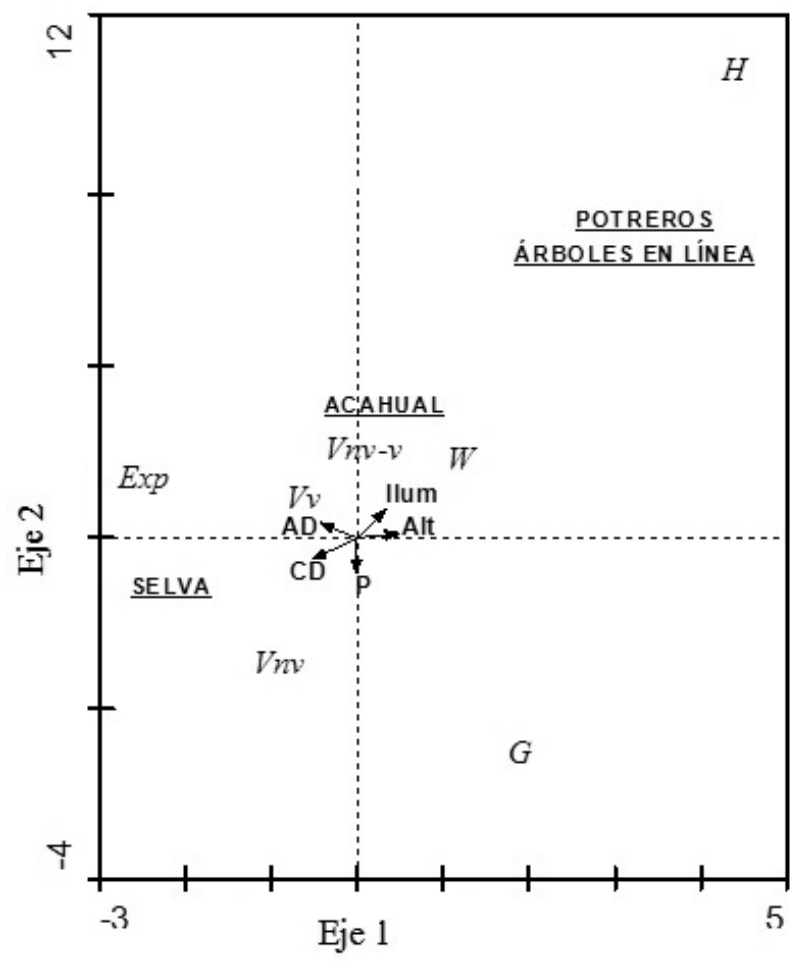

Figura 4. Correspondencia canónica entre mecanismos de dispersión y variables ambientales. $\mathrm{H}$, humanos; Exp, autocoria explosiva; G, autocoria por gravedad; W, anemocoria; Vnv, vertebrado no volador; Vv, vertebrado volador; Vnv-v, vertebrados voladores y no voladores. En el recuadro, las variables ambientales: P, pendiente (\%); Ilum, iluminación (Mjoul $\left./ \mathrm{m}^{2} / \mathrm{año}\right)$; $\mathrm{CD}$, cobertura de dosel (\%); AD, altura media del dosel (m); Alt, altura sobre el nivel del mar (m snm). Gradiente de perturbación de más estable (izquierda) a más perturbado (derecha).

registran Williams-Linera y Lorea (2009), incluyendo dap de $>2.5 \mathrm{~cm}$, mientras que el resultado de este estudio es con base en dap $\geq 5$ centímetros. Ochoa-Gaona y Domínguez-Vázquez (2000) registran 192 especies de árboles en Chajul, que es parte de la Selva Lacandona, número similar al total de especies arbóreas registradas en este estudio. Diversidad de especies. Difícilmente podrían compararse los resultados obtenidos aquí con los de otros estudios debido a las diferencias metodológicas que presentan las investigaciones y las técnicas de muestreo aplicados por los distintos autores (Cuadro 4). No obstante, puede considerarse que el número de especies registradas para el paisaje en estudio es relativamente similar al encontrado en otros de la flora arbórea neotropical. En Belice se encontró una riqueza de 33 a 43 especies arbóreas con dap $\geq 5$ en 4 ambientes diferentes identificados dentro de selva húmeda utilizando transectos de 0.1 ha, lo que resulta aproximado a los datos de áreas iguales en el presente estudio (Brewer et al., 2003). 


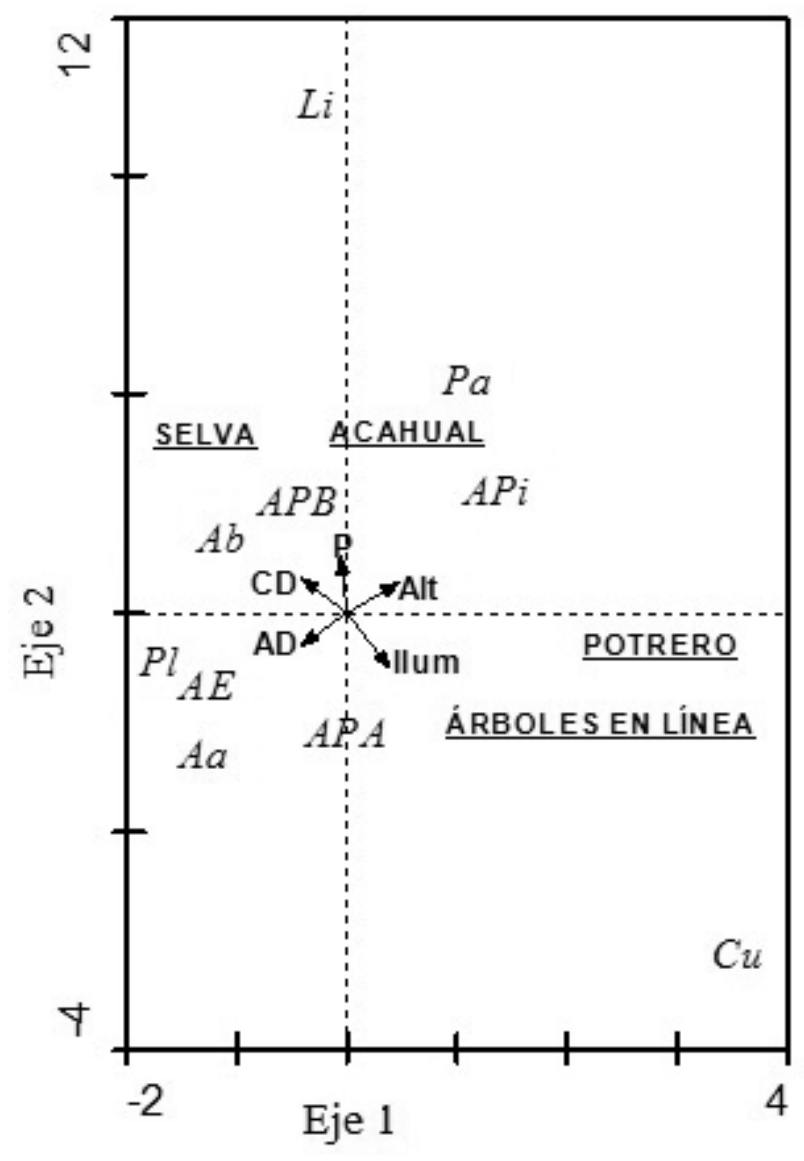

Figura 5. Correspondencia canónica entre los grupos funcionales de la flora arborescente y variables ambientales. Grupos funcionales: Aa, arbustivo alto; $\mathrm{Ab}$, arbustivo bajo; $\mathrm{AE}$, árbol emergente; APA, árbol persistente alto; APB, árbol persistente bajo; $\mathrm{APi}$, árbol pionero; $\mathrm{Cu}$, árbol cultivado; $\mathrm{Li}$, liana; $\mathrm{Pa}$, pionero arbustivo; Pl, palma. En el recuadro, las variables ambientales: $\mathrm{P}$, pendiente (\%); Ilum, iluminación (Mjoul $/ \mathrm{m}^{2} / \mathrm{año}$ ); CD, cobertura de dosel (\%); AD, altura media del dosel (m); Alt, altitud (m). Gradiente de perturbación de menos (izquierda) a más perturbado (derecha).

Al analizar la diversidad y riqueza de especies arbóreas en las unidades matriz-selva en pares, se observa un contraste gradual de acahuales maduros a pastizales con su referente ecológico, encontrando especialmente extremas las unidades de selva con las de los pastizales; lo cual indica un gradiente de perturbación y capacidad de resiliencia de las especies arbóreas (Ochoa-Gaona et al., 2007). Algo similar se observa al analizar el sistema como un todo; se percibe una dinámica entre sus elementos que en conjunto mantienen un continuo entre los ecomosaicos de matriz y selva que comparten un $50 \%$ de su ensamble. La riqueza de especies entre las unidades difiere como un resultado directo del manejo agropecuario de la finca o parcela ejidal, lo que coincide con estudios conducidos a nivel de unidad de manejo o subsistema productivo (Guevara et al., 1992; Harvey y Haber, 1999; Schulze et al., 2004; Schroth et al., 2005). En este estudio, al considerar de manera integral todo el paisaje, que incluye áreas de conservación y de manejo -que corresponde a la realidad en localidades rurales de América-, puede observarse que la matriz agropecuaria se complementa y enriquece por los remanentes de selva, mostrando con ello su capacidad de sostener alta diversidad arbórea, como sugieren Vandemeer y Perfecto (2007). Lo anterior se confirma observando los altos valores obtenidos para el índice de Shannon-Wiener en todas las unidades de la matriz de manera independiente y comparando los pares matriz-selva (Ramírez-González, 2006), superiores a los registrados por Pérez et al. (2005) en agropaisajes del trópico húmedo y similares a los de Godínez-Ibarra y López-Mata (2002), Tun-Dzul (2007), Castro-Luna (2007) y Zamora-Crescencio et al. (2008) para selvas tropicales del sur y sureste de México. El índice Simpson muestra que no hay dominancia de especies, lo cual junto con la elevada equitatividad de las comunidades arbóreas indica que las especies están representadas por abundancias similares dentro de las unidades de paisaje del ejido. Los acahuales incrementan la diversidad de especies de la matriz, pero ésta se mantiene como un grupo diferenciado de la comunidad arbórea característica de la selva, por lo que resulta imprescindible mantener la selva remanente como reserva ejidal dentro del paisaje.

Estructura. Las diferencias estructurales que se encontraron entre las unidades del paisaje, se deben en gran medida al efecto de intensidad de disturbio que se manifiesta extrema entre las unidades de potreros con árboles dispersos y las de selva. El área basal y las densidades encontradas en la selva y acahuales son comparables a las que presentan las selvas y vegetación secundaria en la Amazonía (Vieira et al., 2003), los bosques neotropicales montanos en Bolivia (Araujo-Murakami et al., 2005), los de tierras bajas en Ecuador (Korning y Balslev, 1994) o los obtenidos en la Selva Lacandona por Ochoa-Gaona et al. (2007). Las unidades de vegetación secundaria o acahuales maduros son elementos del paisaje intermedios y permiten una secuencia entre los 2 ecomosaicos (Martin et al., 2004). La estructura de estos acahuales maduros, genera condiciones básicas de cobertura y altura de la vegetación que facilitan la colonización, el establecimiento de plántulas y el desarrollo de juveniles de las especies leñosas nativas del interior de la selva (OchoaGaona et al., 2007).

Composición. La composición de las comunidades arbóreas aún logra sostener una flora nativa diversa a escala de paisaje. Al comparar en pares las unidades del paisaje de la matriz agropecuaria respecto a las unidades de la 
Cuadro 3. Lista de las 27 especies arbóreas que caracterizan las unidades del paisaje y los ecomosaicos en el ejido Niños Héroes de Chapultepec, Tenosique, Tabasco. Las cifras expresan el aporte porcentual de las especies más relevantes para cada unidad que acumulan juntas más del $30 \%$ de la similitud total

\begin{tabular}{|c|c|c|c|c|c|c|c|c|}
\hline Especie característica & $R E_{P A D}$ & $P A D$ & $R E L$ & $A L$ & $R E_{A J}$ & $A J$ & $R E_{A M}$ & $A M$ \\
\hline Tabebuia rosea & & 37.5 & & 6.1 & & & & \\
\hline Byrsonima crassifolia & & 32.5 & & 1.8 & & & & \\
\hline Guazuma ulmifolia & & 11.8 & & 5.2 & & & & \\
\hline Spondias mombin & & 10.5 & & 4.5 & & 4.8 & & \\
\hline Pimienta dioica & & & & 6.7 & & 1.1 & & \\
\hline Swietenia macrophylla & & & & 11.8 & & & & \\
\hline Cedrela odorata & & & & 5.9 & & & & \\
\hline Lippia cardiostegia & & & 3.4 & 16.7 & & 17.6 & & 7.9 \\
\hline Heliocarpus donnell-smithii & & & 3.7 & & & 17.3 & & 9.7 \\
\hline Myriocarpa longipes & & & & & & 1.5 & & 6.4 \\
\hline Trichospermum mexicanum & & & & & & 9.7 & & 3.5 \\
\hline Rhedera penninervia & & & & & & 5.2 & & 8.2 \\
\hline Cecropia obtusifolia & & & 2.6 & & & 4.9 & & \\
\hline Acacia mayana & & & & & 1.1 & 2.9 & & 4.5 \\
\hline Bursera simaruba & & & & & & 3.1 & & 2.0 \\
\hline Mortoniodendron guatemalense & & & & & 1.0 & & & 4.5 \\
\hline Cryosophila argentea & 4.6 & & 2.4 & & 4.5 & & 5.2 & 6.1 \\
\hline Pseudolmedia oxyphyllaria & 4.5 & & 2.1 & & 4.2 & & 5.6 & 4.3 \\
\hline Sebastiania tuerckheimiana & 4.4 & & 11.5 & & 4.3 & & 3.5 & \\
\hline Rinorea guatemalensis & 7.1 & & 7.2 & & 6.3 & & 8.0 & \\
\hline Pouteria campechiana & 3.8 & & 7.1 & & 2.3 & & 4.3 & \\
\hline Quararibea funebris & 2.4 & & 2.9 & & 4.2 & & 4.1 & \\
\hline Chionanthus oblanceolatus & 6.1 & & 0.9 & & 4.2 & & 5.8 & \\
\hline Protium copal & 3.1 & & 0.8 & & 2.1 & & 3.4 & \\
\hline Pouteria reticulata & 1.4 & & & & 5.2 & & 5.4 & \\
\hline Aspidosperma megalocarpon & 5.1 & & & & 2.9 & & 1.3 & \\
\hline Eugenia sp. & & & & & 2.4 & & 3.7 & \\
\hline
\end{tabular}

Unidades de paisaje: AM, acahual maduro; $\mathrm{AJ}$, acahual joven; $\mathrm{AL}$, árboles en línea; $\mathrm{PAD}$, potrero con árboles dispersos; $\mathrm{RE}_{\mathrm{AM}}$, selva referente de los acahuales maduros; $\mathrm{RE}_{\mathrm{AJ}}$; selva referente de los acahuales jóvenes; $\mathrm{RE}_{\mathrm{PAD}}$, selva referente a potreros; $\mathrm{REL}$, elementos de perturbación lineal dentro de la selva o senderos.

selva, se encuentra que éstas difieren en sus ensambles arbóreos de manera notable; sin embargo, es importante notar que las unidades de selva comparten un $40 \%$ de su ensamble con los acahuales, lo que puede ser producto tanto de la perturbación humana dentro de la selva como del papel de los acahuales maduros que con el tiempo permiten el establecimiento de una proporción importante de las especies arbóreas nativas, lo cual es posible por la fuente de germoplasma disponible en los cercanos remanentes de selva. En este sentido, Ochoa-Gaona et al. (2007) indican que a mayor tiempo de desarrollo de la vegetación secundaria sobre las tierras bajo uso agropecuario, mayor es la capacidad de los acahuales para permitir el establecimiento de especies nativas con más altos requerimientos ecológicos (humedad y sombra). Es por ello que alrededor del $50 \%$ de las especies se comparten entre la matriz y la selva; y sólo un $25 \%$ se encontró en cada uno de los ecomosaicos.
Las especies del dosel son prácticamente las mismas que señala Wendt (1998) para Tabasco y Chiapas y muy similares a la composición de especies y familias descrita por Sandoval (1999) en el Petén, Guatemala y por Godínez-Ibarra y López-Mata (2002) en diversas selvas del trópico americano. Muchas de estas especies son propias de bosque maduro (Ochoa-Gaona et al., 2007). La composición y diversidad arbórea en el ejido es el resultado de la forma en que éste ha sido manejado y que incluye la conservación de una reserva ejidal de selva que conforma un área compacta y de remanentes de ésta inmersos en la matriz agropecuaria que, a su vez, aún es heterogénea. Los pastizales conservan una proporción importante de especies, debido a que por el manejo se dejan árboles nativos y se siembran árboles dispersos para sombra y otros usos del ganado, y porque los potreros se delimitan mediante cercos vivos en los cuales se registraron el $33 \%$ de las especies, conformando un ensamble arbóreo diferenciado, en el cual 
Cuadro 4. Resultados procedentes de diferentes estudios sobre riqueza de árboles en el trópico incluyendo detalles de la superficie total muestreada y dap considerados

\begin{tabular}{lccccl}
\hline Localidad & dap* & $R A$ & $S M$ & $M M$ & \\
\hline Santa Gertrudis, Vega de la Torre, Ver. & $\geq 1 \mathrm{~cm}$ & 131 & 3.00 & $3(1)$ & Godor \\
Selva Lacandona, Chis. & ND & 178 & ND & ND & Levy-Tacher et al., 2006 \\
Selva Lacandona, Chis. & $\geq 5 \mathrm{~cm}$ & 119 & 3.90 & $39(0.1)$ & Ochoa-Gaona et al., 2007 \\
Sierra de Tenosique, Tab. & $\geq 10 \mathrm{~cm}$ & 75 & 1.25 & $5(0.25)$ & Ochoa-Gaona et al., 2008 \\
Laguna del Tigre, Petén, Gua. & $\geq 10 \mathrm{~cm}$ & 135 & 1.00 & $10(0.1)$ & Sandoval-Cumes, 1999 \\
Tzucacab, Yuc. & $\geq 3 \mathrm{~cm}$ & 128 & 0.90 & $9(0.1)$ & Zamora-Crescencio et al., 2008 \\
Ejido Ceibita, Tacotalpa, Tab. & $\geq 3 \mathrm{~cm}$ & 51 & 0.50 & $1(0.5)$ & Maldonado-Sánchez y Maldonado-Mares, 2010 \\
Sierra de Tenosique, Tab. & $\geq 5 \mathrm{~cm}$ & 172 & 4.80 & $96(0.05)$ & Presente estudio \\
\hline
\end{tabular}

dap, diámetro a la altura del pecho $(1.30 \mathrm{~m})$; RA, riqueza de árboles; SM, superficie de muestreo (ha); MM,: método de muestreo (número de parcelas y entre paréntesis la superficie de las parcelas en ha).

se incluyen especies exóticas (Fig. 3, Apéndice 1). Se ha demostrado que los cercos vivos funcionan como corredores biológicos para mamíferos pequeños, aves y otros organismos (Fahrig y Merriam, 1985, 1994; Opdam et al., 1993; MacDonald, 2003).

Respuesta de grupos funcionales. La proporción de dispersión de las especies arbóreas del ejido: zoocoras (74\%), anemocoras $(17 \%)$, autocoras $(7 \%)$, cultivadas $(2 \%)$, sigue el mismo patrón registrado en vegetación ribereña (Budke et al., 2005), fragmentos de selva (Kinoshita et al., 2006), en sabanas estacionales (Silva et al., 2009), y en la selva cálido-húmeda de Veracruz (Ibarra-Manríquez et al., 1991). Las especies zoocoras se encontraron asociadas a la selva mediana y a las unidades de vegetación secundaria. La zoocoria puede relacionarse con el grado de estabilidad de los hábitats tropicales (Fleming et al., 1987). En el paisaje del ejido, un importante número de especies de masto y ornitofauna está ligado a la flora arbórea. Lo que se evidencia por la abundancia de plántulas y juveniles registrados en la selva, la vegetación secundaria y en algunas líneas de árboles. Por otro lado, mecanismos de dispersión menos dependientes de la fauna, como la anemocoria y la autocoria tienden a ser más frecuentes en las especies presentes en las unidades de la matriz agropecuaria con mayor grado de alteración (AL y PAD).

Por tipo de árbol, las especies arbóreas persistentes, las arbóreas emergentes, los arbustos, las palmas y las lianas leñosas (dap $\geq 5 \mathrm{~cm}$ ) que predominan en la selva y acahuales maduros ( 3 a 4 estratos de vegetación) demuestran constancia, persistencia, resiliencia y, por tanto, la estabilidad (Grimm y Wissel, 1997) característica de los bosques primarios (Holling, 1973; Chesson, 2000) favorecida por un manejo agropecuario de baja a moderada intensidad en cerca del $50 \%$ del paisaje bajo estudio, lo que facilita, según Yepes et al.(2010), la restitución estructural arbórea.

Las especies pioneras predominan en unidades de árboles en línea y en potreros, pero también están presen- tes en las veredas dentro de la selva y muestran el efecto de la perturbación humana más intensa sobre el ensamble original, que inicia procesos de sucesión vegetal similares a los que ocurren dentro de los claros de la selva (Brokaw, 1985; Fahey y Puettmann, 2007).

Consideraciones generales. La diversidad de árboles en el paisaje estudiado es producto del manejo y sus ecomosaicos muestran complementariedad. El que la matriz contenga un número de especies similar al de la selva y el que se compartan casi el 50\% de las especies está relacionado con el hecho de que los campesinos mantienen áreas de reserva de selva, tanto como un núcleo compacto como con fragmentos distribuidos dentro del paisaje. Esto permite que las parcelas de uso agropecuario queden adyacentes a aquellas y por dispersión sean enriquecidas con especies arbóreas propias de los bosques. Los acahuales durante su desarrollo van generando condiciones básicas de cobertura y altura de la vegetación que facilitan la colonización, el establecimiento de plántulas y el desarrollo de juveniles de las especies leñosas nativas del interior de la selva (Ochoa-Gaona et al., 2007). Por otra parte, el manejo de los potreros incluye la permanencia de árboles remanentes y el establecimiento de cercos vivos que contienen especies propias de las selvas enriquece el paisaje, como sugieren Guevara et al. (1992) y Jobbágy et al. (2006).

No hay que perder de vista que los productores tienen que hacer uso de los recursos para su sobrevivencia; en este sentido, la estrategia para la sustentabilidad debe buscar que se concilie la conservación de especies con el manejo productivo (Holling, 2001; Martínez, 2003). Para ello, como sucede hasta ahora en el ejido, el manejo del paisaje debe mantener a través del tiempo una proporción de hábitats conservados y de remanentes dispersos en el paisaje que enriquezcan la matriz agropecuaria. Asimismo, la matriz debe mantenerse diversificada y conservar los elementos arbóreos, idealmente mediante prácticas agroforestales que se reflejen, a la vez, en una 
mejoría del bienestar de las familias campesinas. El paisaje estudiado nos indica que por ahora, las áreas productivas tienen capacidad de conservar un buen número de especies, llegando al manejo óptimo que se aproximaría al descrito por Diemont et al. (2006) dentro de la Selva Lacandona.

En la zona de estudio, el sistema de acahuales tiende a desaparecer. Por un lado, en las zonas de mayor pendiente algunos productores planean abandonar esta práctica y permitir el desarrollo de los acahuales indefinidamente, lo que favorecería las áreas forestales. Sin embargo, la mayoría de los productores pretenden transformarlos en pasturas, lo que homogeneizará la matriz y aumentará el contraste entre ecomosaicos, impidiendo la dispersión, el establecimiento y el mantenimiento de la fracción de flora arbórea nativa que persiste en estos hábitats, dificultando la conservación de las especies asociadas al mismo (Quintana-Ascencio et al., 1996; Moya-García et al., 2003; Vieira et al., 2009).

\section{Agradecimientos}

A los ejidatarios de Niños Héroes de Chapultepec, Tenosique por su colaboración, en especial a Vicente López Moreno, todo un parataxónomo. A Miguel Martínez Icó, por su invaluable apoyo en la determinación taxonómica. A los herbarios de ECOSUR-San Cristóbal de las Casas y de la UJAT, en especial a Mario Ishiki-Ishihara, Silvia Capello-García y Ángeles Guadarrama-Olivera, por facilitarnos el acceso a las colecciones. Alfonso ReyesDíaz y Jaime Haas-Tzuc apoyaron en la toma de datos en campo. FOMIX-CONACYT-Tabasco financió este trabajo bajo el proyecto Especies forestales de uso múltiple: caracterización de sus usos y potencial de aplicación en planes de fomento forestal, agroforestal y silvopastoril en el municipio de Tenosique, Tabasco, TAB-2007-C09-74820. A ECOSUR, por el apoyo logístico y de infraestructura. A la DIA-SRE, por la beca 811-06-3-728.5/6457/07 para estudios de posgrado del primer autor.

\section{Literatura citada}

Alcázar, C. 2007. Patrones de regeneración natural establecida de especies leñosas y su relación con aspectos estructurales, funcionales y de manejo en un agropaisaje mesoamericano. Tesis, maestría CATIE, Turrialba.196 p.

Araujo-Murakami, A., P. M. Jørgensen, C. Maldonado y N. Paniagua-Zambrana. 2005. Composición florística y estructura del bosque de ceja de monte en Yungas, sector de Tambo Quemado-Pelechuco, Bolivia. Ecología en Bolivia 40:325-338.

Box, G. y D. Cox. 1964. An analysis of transformations. Journal of Research of the Statistical Society 26:211-243.

Brewer, S.W., M. Rejmánek, M. A. H. Webb y P. V. A. Fine. 2003. Relationships of phytogeography and diversity of tropical tree species with limestone topography in southern Belize. Journal of Biogeography 30:1669-1688.

Brokaw, N. V. L. 1985. Gap-phase regeneration in a tropical forest. Ecology 66:682-687.

Budke, J. C., E. Anversa, E. Hettwer, R. Aquino y S. Eisinger. 2005. Composição florística e estratégias de dispersão de espécies lenhosas em uma floresta ribeirinha, arroio Passo das Tropas, Santa Maria, RS, Brasil. Iheringia-Série Botánica 60:17-24.

Castro-Luna, A. A. 2007. Cambios en los ensamblajes de murciélagos en un paisaje modificado por actividades humanas en el trópico húmedo de México. Tesis, doctorado Instituto de Ecología, Xalapa, Veracruz. 119 p.

Chesson, P. L. 2000. Mechanisms of maintenance of species diversity. Annual Review of Ecology and Systematics 31:343-366.

Clarke, K. R. y R. N. Gorley. 2006. Primer v.6: User manual/ Tutorial. Plymouth routine in multivariate ecological research (Primer-e Ltd.). Plymouth. 182 p. http://www.primer-e.com; última consulta: 16.VII.2008.

Clench, H. 1978. To make regional lists of butterflies some thoughts. Lepidopterists Society 33:216-231.

Colwell, R. K. 2006. EstimateS: statistical estimation of species richness and shared species from samples, ver. 8. Persistent URL<purl.oclc.org/estimates>;última consulta: 12.VI.2008.

Diemont, S. A. W., J. F. Martin, S. I. Levy-Tacher, R. B. Nigh, P. R. López y J. D. Golicher. 2006. Lacandon Maya forest management: Restoration of soil fertility using native tree species. Ecological Engineering 28:205-212.

Escobar-Ocampo, M. y S. Ochoa-Gaona. 2007. Estructura y composición florística de la vegetación del Parque Educativo Laguna Bélgica, Chiapas, México. Revista Mexicana de Biodiversidad 78:391-419.

Fahey, R. T. y K. J. Puettmann. 2007. Ground-layer disturbance and initial conditions influence gap partitioning of understorey vegetation. Journal of Ecology 95:1098-1109.

Fahrig, L. y G. Merriam. 1985. Habitat patch connectivity and population survival. Ecology 66:1762-1768.

Fahrig, L. y G. Merriam. 1994. Conservation of fragmented populations. Conservation Biology 8:50-59.

Fleming, T. H., R. Breitwisch y G. H. Whitesides. 1987. Patterns of tropical vertebrate frugivore diversity. Annual Review of Ecology, Evolution and Systematics 18:91-109.

Gobierno del Estado de Tabasco. 1997. Municipio de Tenosique. Secretaría de Desarrollo Social y Protección Ambiental, Gobierno del Estado de Tabasco. Villahermosa. 23 p.

Godínez-Ibarra, O. y L. López-Mata. 2002. Estructura, composición, riqueza y diversidad de árboles en tres muestras de selva mediana perennifolia. Anales del Instituto 
de Biología. Universidad Nacional Autónoma de México, Serie Botánica 73:283-314.

Grimm, V. y C. Wissel. 1997. Babel, or the ecological stability discussions: an inventory and analysis of terminology and guide for avoiding confusion. Oecologia 109:323-334.

Guevara, S., J. Meave, P. Moreno-Casasola y J. Laborde. 1992. Floristic composition and structure of vegetation under isolated trees in neotropical pasture. Journal of Vegetation Science 3:65-664.

Gutiérrez, D., J. León-Cortés, R. Menéndez, R. Wilson, J. Cowley y C. Thomas. 2001. Metapopulations of four lepidopteran herbivores on a single host plant, Lotus corniculatus. Ecology 82:1371-1386.

Habit, E., P. Victoriano y A. Rodríguez-Ruiz. 2003. Variaciones espacio-temporales del ensamble de peces de un sistema fluvial de bajo orden del centro-sur de Chile. Revista Chilena de Historia Natural 76:3-14.

Hammer, Ø., D. Harper y P. Ryan. 2001. Past: Paleontological Statistics Software Package for education and data analysis. Paleontología Electrónica 4: 1-9. http://palaeoelectronica.org/2001_1/past/issue1_01.htm; última consulta: 14.VII.2008.

Harvey, C. y W. Haber. 1999. Remnant trees and the conservation of biodiversity in Costa Rican pastures. Agroforestry Systems 44:37-68.

Harvey, C., O. Komar, R. Chazdon, B. Ferguson, B. Finegan, D. Griffith, M. Martínez-Ramos, H. Morales, R. Nigh, L. Soto-Pinto, M. Van Breugel y M. Wishnie. 2008. Integrating agricultural landscapes with biodiversity conservation in the Mesoamerican hotspot. Conservation Biology 22:8-15.

Hernández-Daumás, S. 2005. La frontera de colonización asistida. La ganadería bovina en la frontera de reciente colonización: el caso de Balancán y Tenosique, Tabasco. In Frontera Sur de México: cinco formas de interacción entre sociedad y ambiente, S. Hernández-Daumás (coord.). El Colegio de la Frontera Sur, Tapachula, Chiapas. p 89-97.

Herrando-Pérez, S. 2002. Manual de ecología matemática. Un enfoque práctico al análisis multivariado (PCA, CLUSTER y MDS) para detectar patrones en ecología. Manual de Referencia, segunda edición. ECOSUR, Chetumal, Quintana Roo. 60 p.

Higgs, E. 2003. Nature for design: People, natural processes and ecological restoration. Massachusetts Institute of Technology, Massachusetts. 57 p.

Holling, C. S. 1973. Resilience and stability of ecological systems. Annual Review of Ecology and Systematics 4:1-23.

Holling, C. S. 2001. Understanding the complexity of economic, ecological and social systems. Ecosystems 4:390-405.

Ibarra-Manríquez, G., B. Sánchez-Garfias y L. González-García. 1991. Fenología de lianas y árboles anemocoros en una selva cálido-húmeda de México. Biotropica 23:242-254.

Isaac-Márquez, F., B. de Jong, A. Eastmond, S. Ochoa-Gaona y
S. Hernández. 2005. Estrategias productivas campesinas: un análisis de los factores condicionantes del uso de suelo en el Oriente de Tabasco, México. Universidad y Ciencia 21:5672.

Isaac-Márquez, F., B. de Jong, A. Eastmond, S. OchoaGaona, S. Hernández y J. L. Sandoval. 2008. Programas gubernamentales y respuestas campesinas en el uso del suelo: El caso de la zona oriente de Tabasco, México. Región y Sociedad 20:97-129.

Jobbágy, E. G., M. Vasallo, K. A. Farley, G. Piñeiro, M. F. Garbulsky, M. D. Nosetto, R. B. Jackson y J. M. Paruelo. 2006. Forestación en pastizales: Hacia una visión integral de sus oportunidades y costos ecológicos. Agrociencia 10:109124.

Kinoshita, L. S., R. Buzanelli-Torres, E. R. Forni-Martin, T. Spinelli, Y. J. Ahn y S. Silva-Constâncio. 2006. Composição florística e síndromes de polinização e de dispersão da mata do Sìtio São Francisco, Campinas, SP, Brasil. Acta Botanica Brasilica 20:313-327.

Korning, J. y H. Balslev. 1994. Growth and mortality of trees in Amazonian tropical rain forest in Ecuador. Journal of Vegetation Science 5:77-86.

Lepš, J. y P. Šmilauer. 2005. Multivariate analysis of ecological data using CANOCO. Cambridge University Press. 269 p.

Levy-Tacher, S. I., J. R. Aguirre Rivera, J. D. García Pérez y M. M. Martínez Romero. 2006. Aspectos florísticos de Lacanhá Chansayab, Selva Lacandona, Chiapas. Acta Botanica Mexicana 77:69-98.

Magurran, A. E. 1988. Ecological diversity and its measurement. Princeton University Press, New Jersey. 179 p.

Maldonado-Sánchez, E. A. y F. Maldonado-Mares. 2010. Estructura y diversidad arbórea de una selva alta perennifolia en Tacotalpa, Tabasco, México. Universidad y Ciencia 26:235-245.

MacDonald, M. A. 2003. The role of corridors in biodiversity conservation in production forest landscapes: a literature review. Tasforests 14:41-52.

Martin, P., R. E. Sherman y T. J. Fahey. 2004. Forty year of tropical forest recovery from agriculture: structure and floristics of secondary and old-growth riparian forests in the Dominican Republic. Biotropica 36:297-317.

Martínez, D. 2003. Protected areas, indigenous peoples and the western idea of nature. Ecological Restoration 21: 247-250.

Masera, O., M. Astier y S. López-Ridaura. 1999. Sustentabilidad y manejo de los recursos naturales: El marco de la evaluación MESMIS. Mundi-Prensa, México, D.F. 109 p.

Morrison, E. 1999. Introduction to SPSS. Center for Social Science Computation and Research, Universidad de Washington. $14 \mathrm{p}$.

Moya-García, X., A. Caamal, B. Ku-Ku, E. Chan-Xool, I. Armendáriz, J. Flores, J. Moguer, M. Noh-Poot, M. Rosales y J. Xool-Domínguez. 2003. La agricultura campesina de los 
mayas en Yucatán. LEISA Revista de Agroecología 19:7-17.

Ochoa-Gaona, S. y G. Domínguez-Vázquez. 2000. Distribución y fenología de la flora leñosa de Chajul, Selva Lacandona, Chiapas, México. Brenesia 54:1-24.

Ochoa-Gaona, S., M. González-Espinosa, J. Meave y V. SoraniDal Bon. 2004. Effect of forest fragmentation on the woody flora of highlands of Chiapas, Mexico. Biodiversity and Conservation 13:867-884.

Ochoa-Gaona, S., F. Hernández-Vázquez, B. H. J. De Jong y F. Gurri-García. 2007. Pérdida de diversidad florística ante un gradiente de intensificación del sistema agrícola de rozatumba-quema: un estudio de caso en la Selva Lacandona, Chiapas, México. Boletín de la Sociedad Botánica de México 81:65-80.

Ochoa-Gaona, S., I. Pérez-Hernández y B. De Jong. 2008. Fenología reproductiva de las especies arbóreas del bosque tropical de Tenosique, Tabasco, México. Revista de Biología Tropical 56:657-673.

Opdam, P., R. van Apeldoorn, A. Schotman y J. Kalkhoven. 1993. Population responses to landscape fragmentation. In Landscape ecology of a stressed environment, C. C. Vos y P. Opdam (eds.). Chapman and Hall, NewYork. p. 147-177.

Ortiz-Espejel, B. y V. Toledo. 1998. Tendencias en la deforestación de la Selva Lacandona (Chiapas, México): el caso de Las Cañadas. Interciencia 23:318-327.

Pérez, A. M., G. Borneman, L. Campo, M. Sotelo, F. Ramírez e I. Arana. 2005. Relaciones entre biodiversidad y producción en sistemas silvopastoriles de América Central. Ecosistemas 14:132-141.

Pérez, A. M., M. Sotelo, I. Siria, R. Alkemade y L. Aburto. 2007. Developing a species based model for biodiversity assessment in an agricultural landscape in Nicaragua. Gaia $8: 1-54$.

Quintana-Ascencio P. F., M. González-Espinosa, N. RamírezMarcial, G. Domínguez-Vázquez y M. Martínez-Icó 1996. Soil seed banks and regeneration of tropical rain forest from milpa fields at the Selva Lacandona, Chiapas, Mexico. Biotropica 28:192-209.

Ramírez-González, A. 2006. Ecología: métodos de muestreo $\mathrm{y}$ análisis de poblaciones y comunidades. Pontificia Universidad Javeriana, Bogotá. p. 273.

Sánchez, D., C. Harvey, A. Grijalva, A. Medina, S. Vílchez y B. Hernández. 2005. Diversidad, composición y estructura de la vegetación en un agropaisaje ganadero en Matiguás, Nicaragua. Revista de Biología Tropical 53:387-414.

Sandoval-Cumes, K. J. 1999. Análisis estructural de la vegetación arbórea y sotobosque del Parque Nacional Laguna El Tigre, Petén, Guatemala. Tesis, Universidad de San Carlos de Guatemala, Guatemala. 104 p.

Schroth, G., G. Da Fonseca, C. Harvey, C. Gascón, L. Vasconcelos y A. Izac. 2005. Farmers and the forest: can the agroforestry actually conserve biodiversity? Conservation Biology
19:2043-2044.

Schulze, C., M. Waltert, P. Kessler, R. Pitopang, Shahabuddin, D. Veddeler, M. Mühlenberg, R. Gradstein, C. Leuschner, I. Steffan-Dewenter y T. Tscharntke. 2004. Biodiversity indicator groups of tropical land-use system: comparing plants, birds, and insects. Ecological Applications 14:13211333.

SEDESPA (Secretaría de Desarrollo Social y Protección al Ambiente). 2006. Programa de Ordenamiento Ecológico del Estado de Tabasco, Villahermosa. 99 p.

Sekercioglu, C., S. Loarie, F. Oviedo, P. Ehrlich y G. Daily. 2007. Persistence of forest birds in the Costa Rican agricultural countryside. Conservation Biology 21:482-494.

SEMARNAT (Secretaría de Medio Ambiente y Recursos Naturales). 2008. Decreto por el que se declara área natural protegida con la categoría de área de protección de flora y fauna, la región conocida como cañón del Usumacinta, localizada en el municipio de Tenosique, en el estado de Tabasco. Diario Oficial de la Federación, 22 de septiembre de 2008. 16 p. http://vlex.com.mx/vid/categoria-florafauna-usumacinta-tenosique-42835362;última consulta: 23.VIII.2008.

Silva, I. A., M. V. Cianciaruso y M. A. Batalha. 2009. Dispersal modes and fruiting periods in hyperseasonal and seasonal savannas, central Brazil. Revista Brasileira de Botânica 32:155-163.

Smith, T. M., H. H. Shugart y F. I. Woodward. 1998. Plant functional types: their relevance to ecosystem properties and global change. International Geosphere-Biosphere Programme Books Series, Cambridge University Press. 369 p.

Snow, D. W. 1981. Tropical frugivorous birds and their food plants: a world survey. Biotropica 13:1-14.

Spellerberg, I. F. 2005. Monitoring ecological change, segunda edición. Cambridge University Press. 391 p.

Stevens, M. 1996. SER-'95 Indigenous peoples program. Restoration and Management Notes 14:35-38.

Tobar, D., M. Ibrahim y F. Casasola. 2007. Diversidad de mariposas en un paisaje agropecuario del Pacífico Central de Costa Rica. Agroforestería en las Américas 45:58-65.

Toledo, V. 2002. Agroecología, sustentabilidad y reforma agraria: la superioridad de la pequeña producción familiar. Agroecologia e Desenvolvimento Rural Sustentável 3:27-36.

Tun-Dzul, F. J. 2007. La estacionalidad de la selva baja inundable: su análisis mediante percepción remota. Tesis Maestría, El Colegio de la Frontera Sur, Chetumal, Quintana Roo. 96 p.

Vandemeer, J. e I. Perfecto. 2007. The agricultural matrix and a future paradigm for conservation. Conservation Biology 21:274-277.

Vázquez-Negrin, I. y O. Castillo-Acosta. 2007. Heterogeneidad estructural de un remanente de selva alta perennifolia en el ejido Niños Héroes, Tenosique, Tabasco. In Memorias de 
la Semana de Divulgación y Video Científico, Universidad Juárez Autónoma de Tabasco, Villahermosa. p. 503-506.

Vieira, D. L. M., K. D. Holl y F. M. Peneireiro. 2009. Agrosuccessional restoration as a strategy to facilitate tropical forest recovery. Restoration Ecology 17:451-459.

Vieira, I. C. G., A. Silva, E. A. Davidson, T. A. Stone, C. J. Reis y J. B. Guerrero. 2003. Classifying successional forests using Landsat spectral properties and ecological characteristics in eastern Amazonia. Remote Sensing of Environment 87:470481.

Vílchez, S., C. Harvey, D. Sánchez, A. Medina y B. Hernández. 2004. Diversidad de aves en un paisaje fragmentado de bosque seco en Rivas, Nicaragua. Encuentro 4:60-75.

Wendt, T. 1998. Composición, afinidades florísticas y orígenes de la flora arbórea del dosel de los bosques tropicales húmedos de la vertiente mexicana del Atlántico. In Diversidad biológica de México: orígenes y distribución, T. P. Ramamoorthy, R. Bye, A. Lot y J. Fa (comps.). Instituto de Biología, UNAM, México, D.F. p. 581-664.
Williams-Linera, G. y F. Lorea. 2009. Tree species diversity driven by environmental and anthropogenic factors in tropical dry forest fragments of central Veracruz, Mexico. Biodiversity and Conservation, 18:3269-3293.

Yepes, A. P., J. I. Del Valle, S. L. Jaramillo y S. A. Orrego. 2010. Recuperación estructural en bosques sucesionales andinos de Porce (Antioquia, Colombia). Revista de Biología Tropical, 58:427-445.

Zamora-Crescencio, P., G. García, J. Flores y J. Ortiz. 2008. Estructura y composición florística de la selva mediana subcaducifolia en el sur del estado de Yucatán, México. Polibotánica 26:33-66.

Zonneveld, I. 1989. The land unit: a fundamental concept in landscape ecology, and its application. Landscape Ecology 3:67-83.

Zuur, A., E. Ieno y G. Smith. 2007. Analyzing ecological data. In Statistics for biology and health, M. Gail, K. Krickeberg, J. Samet, A. Tsiatis y W. Wong (eds.). Springer Science+Business Media, New York. p. 163-179.

Apéndice 1. Datos de las especies arbóreas con dap $\geq 5 \mathrm{~cm}$ en los ecomosaicos estudiados (la nomenclatura se basa en la página del Missouri Botanical Garden http://www.tropicos.org/).

Claves. FI: fidelidad a hábitats. C, común, que existe en ambos ecomosaicos; MA,sólo en la matriz agropecuaria; RE,sólo en el remanente de selva mediana perennifolia. GF: grupo funcional. Aa, arbustivo alto; Ab, arbustivo bajo; AE, árbol emergente; APA, árbol persistente alto; APB, árbol persistente bajo; APi, árbol pionero; $\mathrm{Cu}$, árbol cultivado; Li, liana; Pa, pionero arbustivo; Pl, palma. MD: mecanismo dispersor. Vnv, vertebrados no voladores principalmente mamíferos; Vv, vertebrados voladores principalmente aves; Vnvv, vertebrados no voladores y voladores; W, anemocoria; G, gravedad; Expl., autocoria explosiva; H, sólo dispersada por humanos. FC: fases de crecimiento. 1 , árboles o arbustos con dap $\geq 5 \mathrm{~cm}$ y altura $\geq 1.5 \mathrm{~m} ; 2$, arbustos o árboles jóvenes $1.5 \mathrm{~cm} \leq$ dap $<5 \mathrm{~cm}$ y altura $<1.5 \mathrm{~m} ; 3$, plántulas. *Especie introducida. No nativa.

\begin{tabular}{|c|c|c|c|c|c|}
\hline Núm. & $\begin{array}{l}\text { Familia } \\
\text { Especies } \\
\text { Actinidiaceae }\end{array}$ & $F I$ & $G F$ & $M D$ & $F C$ \\
\hline 1 & $\begin{array}{l}\text { Saurauia yasicae Loes. } \\
\text { Anacardiaceae }\end{array}$ & $\mathrm{C}$ & APB & $\mathrm{Vv}$ & $1,2,3$ \\
\hline 2 & Astronium graveolens Jacq. & $\mathrm{C}$ & $\mathrm{APi}$ & $\mathrm{Vv}$ & $1,2,3$ \\
\hline 3 & Metopium brownei (Jacq.) Urb. & $\mathrm{C}$ & $\mathrm{APi}$ & Vnv-v & $1,2,3$ \\
\hline 4 & Mosquitoxylum jamaicense Krug et Urb. & $\mathrm{C}$ & APA & Vv & $1,2,3$ \\
\hline 5 & $\begin{array}{l}\text { Spondias mombin } \mathrm{L} \text {. } \\
\text { Annonaceae }\end{array}$ & MA & $\mathrm{APi}$ & Vnv-v & $1,2,3$ \\
\hline 6 & Annona reticulata $\mathrm{L}$. & MA & $\mathrm{Cu}$ & Vnv-v & 1,2 \\
\hline 7 & Cymbopetalum penduliflorum (Dunal) Baill. & $\mathrm{RE}$ & APA & Vv & 1 \\
\hline 8 & Guatteria anomala R.E. Fr. & $\mathrm{RE}$ & $\mathrm{AE}$ & Vv & $1,2,3$ \\
\hline 9 & Oxandra belizensis (Lundell) Lundell & $\mathrm{RE}$ & APA & Vnv-v & $1,2,3$ \\
\hline 10 & Rollinia membranacea Triana et Planch. & $\mathrm{RE}$ & APB & Vnv-v & 1 \\
\hline 11 & $\begin{array}{l}\text { Rollinia mucosa (Jacq.) Baill. } \\
\text { Apocynaceae }\end{array}$ & $\mathrm{C}$ & APB & Vnv-v & 1,2 \\
\hline 12 & Aspidosperma cruentum Woodson & $\mathrm{RE}$ & APA & $\mathrm{W}$ & $1,2,3$ \\
\hline 13 & Aspidosperma megalocarpon Müll. Arg. & $\mathrm{C}$ & APA & W & $1,2,3$ \\
\hline 14 & $\begin{array}{l}\text { Stemmadenia donnell-smithii (Rose) Woodson } \\
\text { Aquifoliaceae }\end{array}$ & $\mathrm{C}$ & APB & Vnv-v & $1,2,3$ \\
\hline 15 & $\begin{array}{l}\text { Ilex costaricensis Donn. Sm. } \\
\text { Araliaceae }\end{array}$ & $\mathrm{C}$ & APA & $\mathrm{Vv}$ & $1,2,3$ \\
\hline
\end{tabular}


Apéndice 1. Continúa

\begin{tabular}{|c|c|c|c|c|c|}
\hline Núm. & $\begin{array}{l}\text { Familia } \\
\text { Especies }\end{array}$ & $F I$ & $G F$ & $M D$ & $F C$ \\
\hline 16 & $\begin{array}{l}\text { Dendropanax arboreus (L.) Decne. et Planch. } \\
\text { Arecaceae }\end{array}$ & $\mathrm{C}$ & $\mathrm{APB}$ & $\mathrm{Vv}$ & $1,2,3$ \\
\hline 17 & Cryosophila argentea Bartlett & $\mathrm{C}$ & $\mathrm{Pl}$ & $\mathrm{Vv}$ & 1,3 \\
\hline 18 & $\begin{array}{l}\text { Geonoma binervia Oerst. } \\
\text { Asteraceae }\end{array}$ & $\mathrm{RE}$ & $\mathrm{Pl}$ & Vnv & $1,2,3$ \\
\hline 19 & $\begin{array}{l}\text { Eupatorium morifolium Mill. } \\
\text { Bignoniaceae }\end{array}$ & MA & $\mathrm{Pa}$ & $\mathrm{W}$ & $1,2,3$ \\
\hline 20 & Parmentiera aculeata (Kunth) Seem. & MA & APi & Vnv & 1,3 \\
\hline 21 & Spathodea campanulata P. Beauv.* & MA & $\mathrm{Cu}$ & $\mathrm{W}$ & $1,2,3$ \\
\hline 22 & Tabebuia donnell-smithii Rose & MA & APB & $\mathrm{W}$ & $1,2,3$ \\
\hline 23 & $\begin{array}{l}\text { Tabebuia rosea (Bertol.) A. DC. } \\
\text { Bombacaceae }\end{array}$ & MA & APi & $\mathrm{W}$ & $1,2,3$ \\
\hline 24 & Ceiba pentandra (L.) Gaertn. & MA & $\mathrm{AE}$ & $\mathrm{W}$ & 1,2 \\
\hline 25 & Pseudobombax ellipticum (Kunth) Dungand & $\mathrm{C}$ & APi & W & $1,2,3$ \\
\hline 26 & $\begin{array}{l}\text { Quararibea funebris (La Llave) Vischer } \\
\text { Boraginaceae }\end{array}$ & $\mathrm{C}$ & APB & $\mathrm{Vv}$ & $1,2,3$ \\
\hline 27 & Cordia alliodora (Ruiz et Pav.) Oken & $\mathrm{C}$ & APA & $\mathrm{W}$ & $1,2,3$ \\
\hline 28 & Cordia dodecandra DC. & RE & APA & Vnv-v & 1 \\
\hline 29 & $\begin{array}{l}\text { Cordia stellifera I.M. Johnst. } \\
\text { Burseraceae }\end{array}$ & $\mathrm{C}$ & APA & $\mathrm{Vv}$ & $1,2,3$ \\
\hline 30 & Bursera instabilis McVaugh et Rzed. & $\mathrm{C}$ & $\mathrm{APi}$ & $\mathrm{Vv}$ & 1,2 \\
\hline 31 & Bursera simaruba (L.) Sarg. & MA & $\mathrm{APi}$ & $\mathrm{Vv}$ & $1,2,3$ \\
\hline 32 & $\begin{array}{l}\text { Protium copal (Schltdl. et Cham.) Eng. } \\
\text { Caricaceae }\end{array}$ & $\mathrm{C}$ & APB & $\mathrm{Vv}$ & $1,2,3$ \\
\hline 33 & $\begin{array}{l}\text { Jacaratia dolichaula (Donn. Sm.) Woodson } \\
\text { Cecropiaceae }\end{array}$ & $\mathrm{C}$ & APi & $\mathrm{Vv}$ & 1,2 \\
\hline 34 & Cecropia obtusifolia Bertol. & $\mathrm{C}$ & APi & Vnv-v & 1,3 \\
\hline 35 & $\begin{array}{l}\text { Coussapoa purpusii Standl. } \\
\text { Celastraceae }\end{array}$ & MA & $\mathrm{APi}$ & $\mathrm{Vv}$ & 1 \\
\hline 36 & $\begin{array}{l}\text { Wimmeria bartlettii Lundell } \\
\text { Chrysobalanaceae }\end{array}$ & $\mathrm{RE}$ & APA & W & 1 \\
\hline 37 & Couepia dodecandra (Moc. et Sessé ex DC) Hemsl. & $\mathrm{C}$ & APA & Vnv & $1,2,3$ \\
\hline 38 & $\begin{array}{l}\text { Licania platypus (Hemsl.) Fritsch. } \\
\text { Clusiaceae }\end{array}$ & MA & APA & Vnv & 1,2 \\
\hline 39 & Calophyllum brasiliense Cambess. & $\mathrm{C}$ & APA & Vnv & 1 \\
\hline 40 & Clusia flava Jacq. & MA & APB & $\mathrm{Vv}$ & 1 \\
\hline 41 & $\begin{array}{l}\text { Garcinia intermedia (Pittier) Hammel } \\
\text { Cochlospermaceae }\end{array}$ & $\mathrm{RE}$ & APB & Vnv-v & $1,2,3$ \\
\hline 42 & $\begin{array}{l}\text { Cochlospermum vitifolium (Willd.) Spreng. } \\
\text { Combretaceae }\end{array}$ & MA & APi & $\mathrm{W}$ & 1 \\
\hline 43 & $\begin{array}{l}\text { Terminalia amazonia (J.F. Gmel.) Exell. } \\
\text { Dracaenaceae }\end{array}$ & $\mathrm{C}$ & $\mathrm{AE}$ & $\mathrm{W}$ & $1,2,3$ \\
\hline 44 & $\begin{array}{l}\text { Dracaena americana Donn. Sm. } \\
\text { Euphorbiaceae }\end{array}$ & $\mathrm{C}$ & APB & $\mathrm{Vv}$ & 1,3 \\
\hline 45 & Acalypha macrostachya Jacq. & MA & $\mathrm{Pa}$ & Vnv & $1,2,3$ \\
\hline 46 & Alchornea latifolia Sw. & $\mathrm{C}$ & APA & $\mathrm{Vv}$ & $1,2,3$ \\
\hline 47 & Cestrum nocturnum L. & $\mathrm{RE}$ & APB & $\mathrm{Vv}$ & 1 \\
\hline 48 & $\begin{array}{l}\text { Sebastiania tuerckheimiana (Pax et K. Hoffm.) Lundell } \\
\text { Fabaceae }\end{array}$ & $\mathrm{C}$ & APB & $\mathrm{Vv}$ & $1,2,3$ \\
\hline 49 & Acacia mayana Lundell & $\mathrm{C}$ & $\mathrm{APi}$ & $\mathrm{Vv}$ & $1,2,3$ \\
\hline 50 & Bauhinia divaricata $\mathrm{L}$. & $\mathrm{RE}$ & APB & $\mathrm{Vv}$ & $1,2,3$ \\
\hline
\end{tabular}


Apéndice 1. Continúa

\begin{tabular}{|c|c|c|c|c|c|}
\hline Núm. & $\begin{array}{l}\text { Familia } \\
\text { Especies }\end{array}$ & $F I$ & $G F$ & $M D$ & $F C$ \\
\hline 51 & Caesalpinia gaumeri Greenm. & MA & $\mathrm{APB}$ & $\mathrm{W}$ & $1,2,3$ \\
\hline 52 & Cojoba arborea (L.) Britton et Rose & MA & $\mathrm{APB}$ & Vv & 1 \\
\hline 53 & Dialium guianense (Aubl.) Sandwith. & $\mathrm{C}$ & $\mathrm{AE}$ & Vnv-v & $1,2,3$ \\
\hline 54 & Erythrina berteroana Urb. & MA & $\mathrm{APi}$ & G & $1,2,3$ \\
\hline 55 & Gliricidia sepium (Jacq.) Kunth ex Walp. & MA & $\mathrm{Cu}$ & G & 1,2 \\
\hline 56 & Haematoxylum campechianum L. & MA & $\mathrm{APi}$ & G & 1,3 \\
\hline 57 & Inga inicuil Schltdl. et Cham. ex G. Don & MA & $\mathrm{APB}$ & Vnv-v & 1 \\
\hline 58 & Inga laurina $(\mathrm{Sw}$.$) Willd.$ & $\mathrm{C}$ & APB & Vnv-v & $1,2,3$ \\
\hline 59 & Inga vera Willd. & $\mathrm{C}$ & APB & Vnv-v & $1,2,3$ \\
\hline 60 & Lonchocarpus castilloi Standl. & $\mathrm{C}$ & APB & G & $1,2,3$ \\
\hline 61 & Lonchocarpus guatemalensis Benth. & $\mathrm{C}$ & $\mathrm{APB}$ & G & $1,2,3$ \\
\hline 62 & Lysiloma acapulcense (Kunth) Benth. & $\mathrm{RE}$ & $\mathrm{APB}$ & W & 1,2 \\
\hline 63 & Lysiloma divaricatum (Jacq.) J.F. Macbr. & MA & $\mathrm{APB}$ & W & 1,3 \\
\hline 64 & Lysiloma latisiliquum (L.) Benth. & $\mathrm{C}$ & $\mathrm{APB}$ & $\mathrm{W}$ & $1,2,3$ \\
\hline 65 & Machaerium biovulatum Micheli & $\mathrm{RE}$ & $\mathrm{Li}$ & W & $1,2,3$ \\
\hline 66 & Machaerium cirrhiferum Pittier. & MA & $\mathrm{APi}$ & W & 1 \\
\hline 67 & Ormosia macrocalyx Ducke & $\mathrm{C}$ & $\mathrm{APB}$ & G & 1 \\
\hline 68 & Ormosia schippii Pierce ex Standl. et Steyerm. & $\mathrm{RE}$ & $\mathrm{APB}$ & G & 1 \\
\hline 69 & Piscidia piscipula (L.) Sarg. & MA & $\mathrm{APi}$ & W & 1,3 \\
\hline 70 & Pithecellobium dulce (Roxb.) Benth. & MA & APi & Vnv-v & 1,3 \\
\hline 71 & Schizolobium parahyba (Vell.) S. F. Blake & $\mathrm{RE}$ & $\mathrm{APi}$ & $\mathrm{W}$ & 1 \\
\hline 72 & Swartzia cubensis (Britton et P. Wilson) Standl. & $\mathrm{C}$ & APA & Vnv-v & $1,2,3$ \\
\hline 73 & Vatairea lundellii (Standl.) Killip ex Record & $\mathrm{C}$ & APA & $\mathrm{W}$ & $1,2,3$ \\
\hline 74 & $\begin{array}{l}\text { Vochysia guatemalensis Donn. Sm. } \\
\text { Flacourtiaceae }\end{array}$ & $\mathrm{RE}$ & APA & W & $1,2,3$ \\
\hline 75 & Casearia commersoniana Cambess. & $\mathrm{RE}$ & APB & Vnv-v & 1,3 \\
\hline 76 & Casearia nitida (L.) Jacq. & MA & $\mathrm{Pa}$ & $\mathrm{Vv}$ & 1 \\
\hline 77 & Casearia sylvestris Sw. & $\mathrm{C}$ & APA & Vv & $1,2,3$ \\
\hline 78 & Casearia tacanensis Lundell. & $\mathrm{C}$ & APB & Vnv-v & $1,2,3$ \\
\hline 79 & $\begin{array}{l}\text { Zuelania guidonia (Sw.) Britton et Millsp. } \\
\text { Lauraceae }\end{array}$ & $\mathrm{C}$ & APA & Vnv-v & $1,2,3$ \\
\hline 80 & Aiouea inconspicua van der Werff & $\mathrm{RE}$ & $\mathrm{APB}$ & $\mathrm{Vv}$ & 1,2 \\
\hline 81 & Cinnamomum grisebachii Lorea-Hern. & $\mathrm{RE}$ & APA & Vnv-v & 1 \\
\hline 82 & Licaria cervantesii (Kunth) Kosterm. & $\mathrm{RE}$ & $\mathrm{APB}$ & $\mathrm{Vv}$ & $1,2,3$ \\
\hline 83 & Nectandra lundellii C.K. Allen & $\mathrm{RE}$ & APB & $\mathrm{Vv}$ & $1,2,3$ \\
\hline 84 & Nectandra salicifolia (Kunth) Nees & $\mathrm{C}$ & APB & $\mathrm{Vv}$ & $1,2,3$ \\
\hline 85 & Ocotea helicterifolia (Meins.) Hemsl. & $\mathrm{RE}$ & $\mathrm{APB}$ & $\mathrm{Vv}$ & $1,2,3$ \\
\hline 86 & $\begin{array}{l}\text { Persea americana Mill. } \\
\text { Magnoliaceae }\end{array}$ & MA & $\mathrm{Cu}$ & Vnv-v & 1,2 \\
\hline 87 & $\begin{array}{l}\text { Talauma mexicana (DC.) Don } \\
\text { Malpighiaceae }\end{array}$ & MA & APB & Vnv-v & $1,2,3$ \\
\hline 88 & Bunchosia sp. & MA & $\mathrm{Pa}$ & $\mathrm{Vv}$ & $1,2,3$ \\
\hline 89 & $\begin{array}{l}\text { Byrsonima crassifolia (L.) Kunth } \\
\text { Malvaceae }\end{array}$ & MA & $\mathrm{Cu}$ & Vnv-v & 1,2 \\
\hline 90 & $\begin{array}{l}\text { Hampea stipitata } \mathrm{S} \text {. Watson } \\
\text { Melastomataceae }\end{array}$ & $\mathrm{C}$ & APi & Vv & $1,2,3$ \\
\hline 91 & Miconia argentea (Sw.) DC. & $\mathrm{RE}$ & $\mathrm{Aa}$ & $\mathrm{Vv}$ & $1,2,3$ \\
\hline 92 & $\begin{array}{l}\text { Miconia impetiolaris (Sw.) D. Don. ex DC. } \\
\text { Meliaceae }\end{array}$ & $\mathrm{RE}$ & Aa & $\mathrm{Vv}$ & $1,2,3$ \\
\hline 93 & Cedrela odorata L. & MA & APA & W & $1,2,3$ \\
\hline 94 & Guarea glabra Vahl. & $\mathrm{C}$ & APA & $\mathrm{Vv}$ & $1,2,3$ \\
\hline
\end{tabular}


Apéndice 1. Continúa

\begin{tabular}{|c|c|c|c|c|c|}
\hline Núm. & $\begin{array}{l}\text { Familia } \\
\text { Especies }\end{array}$ & $F I$ & $G F$ & $M D$ & $F C$ \\
\hline 95 & Guarea petenensis Coronado & $\mathrm{RE}$ & APA & Vv & 1 \\
\hline 96 & $\begin{array}{l}\text { Swietenia macrophylla King. } \\
\text { Monimiaceae }\end{array}$ & $\mathrm{C}$ & APA & W & $1,2,3$ \\
\hline 97 & Siparuna andina (Tul.) A. DC. & $\mathrm{C}$ & $\mathrm{APi}$ & Vv & $1,2,3$ \\
\hline 98 & $\begin{array}{l}\text { Siparuna guianensis Aubl. } \\
\text { Moraceae }\end{array}$ & $\mathrm{C}$ & $\mathrm{Ab}$ & $\mathrm{Vv}$ & $1,2,3$ \\
\hline 99 & Brosimum alicastrum $\mathrm{Sw}$. & $\mathrm{C}$ & APA & Vnv-v & $1,2,3$ \\
\hline 100 & Brosimum lactescens (S. Moore) C.C. Berg. & $\mathrm{C}$ & APA & Vnv-v & $1,2,3$ \\
\hline 101 & Ficus benjamina L.* & MA & $\mathrm{Cu}$ & Vv & 1 \\
\hline 102 & Ficus cotinifolia Kunth & $\mathrm{C}$ & APB & Vv & 1 \\
\hline 103 & Ficus glabrata Kunth & MA & $\mathrm{APB}$ & Vv & 1 \\
\hline 104 & Ficus insipida Willd. & $\mathrm{C}$ & APA & Vnv-v & 1 \\
\hline 105 & Ficus pertusa L. f & $\mathrm{C}$ & $\mathrm{APB}$ & Vv & $1,2,3$ \\
\hline 106 & Ficus petiolaris Kunth & MA & $\mathrm{APB}$ & Vv & 1,3 \\
\hline 107 & Ficus tecolutensis (Liebm.) Miq. & MA & APA & Vnv-v & $1,2,3$ \\
\hline 108 & Poulsenia armata (Miq.) Standl. & $\mathrm{RE}$ & APA & Vnv-v & $1,2,3$ \\
\hline 109 & Pseudolmedia oxyphyllaria Donn. Sm. & $\mathrm{C}$ & $\mathrm{APB}$ & Vnv-v & $1,2,3$ \\
\hline 110 & $\begin{array}{l}\text { Trophis racemosa (L.) Urb. } \\
\text { Myrtaceae }\end{array}$ & $\mathrm{RE}$ & APB & Vv & $1,2,3$ \\
\hline 111 & Eugenia biflora (L.) DC. & $\mathrm{C}$ & $\mathrm{APB}$ & Vv & $1,2,3$ \\
\hline 112 & Eugenia capuli (Schldl. et Cham.) Hook. et Arn. & MA & Aa & Vv & $1,2,3$ \\
\hline 113 & Eugenia capulioides Lundell & MA & Aa & Vv & 1 \\
\hline 114 & Eugenia sp. & $\mathrm{C}$ & APB & $\mathrm{Vv}$ & $1,2,3$ \\
\hline 115 & Pimienta dioica (L.) Merr. & $\mathrm{C}$ & $\mathrm{APB}$ & Vv & $1,2,3$ \\
\hline 116 & Psidium guajava L. & MA & $\mathrm{APi}$ & Vnv-v & $1,2,3$ \\
\hline 117 & $\begin{array}{l}\text { Psidium sartorianum (O. Berg.) Nied. } \\
\text { Ochnaceae }\end{array}$ & $\mathrm{RE}$ & APA & Vnv-v & 1 \\
\hline 118 & $\begin{array}{l}\text { Ouratea lucens (Kunth) Engl. } \\
\text { Olacaceae }\end{array}$ & $\mathrm{RE}$ & APB & Vv & $1,2,3$ \\
\hline 119 & $\begin{array}{l}\text { Ximenia americana } \mathrm{L} \text {. } \\
\text { Oleaceae }\end{array}$ & MA & $\mathrm{Aa}$ & Vv & $1,2,3$ \\
\hline 120 & $\begin{array}{l}\text { Chionanthus oblanceolatus (B.L. Rob.) P.S. Green } \\
\text { Piperaceae }\end{array}$ & $\mathrm{RE}$ & $\mathrm{Aa}$ & Vnv-v & $1,2,3$ \\
\hline 121 & $\begin{array}{l}\text { Piper amalago L. } \\
\text { Rosaceae }\end{array}$ & $\mathrm{C}$ & $\mathrm{Aa}$ & Vnv & $1,2,3$ \\
\hline 122 & $\begin{array}{l}\text { Prunus occidentalis Sw. } \\
\text { Rubiaceae }\end{array}$ & $\mathrm{RE}$ & APA & Vv & 1,2 \\
\hline 123 & Alseis yucatanensis Standl. & $\mathrm{C}$ & APB & Vv & $1,2,3$ \\
\hline 124 & Blepharidium mexicanum Standl. & $\mathrm{C}$ & Aa & Vv & 1 \\
\hline 125 & Chione chiapasensis Standl. & $\mathrm{RE}$ & APA & Vnv-v & $1,2,3$ \\
\hline 126 & Faramea occidentalis (L.) A. Rich. & $\mathrm{RE}$ & $\mathrm{Aa}$ & Vv & $1,2,3$ \\
\hline 127 & Gonzalagunia panamensis (Cav.) K. Schum. & $\mathrm{C}$ & $\mathrm{APi}$ & Vv & $1,2,3$ \\
\hline 128 & Guettarda combsii Urb. & $\mathrm{C}$ & Aa & $\mathrm{Vv}$ & 1 \\
\hline 129 & Hamelia patens Jacq. & MA & $\mathrm{Ab}$ & $\mathrm{Vv}$ & $1,2,3$ \\
\hline 130 & Posoqueria latifolia (Rudge) Roem. et Schult. & $\mathrm{C}$ & APB & $\mathrm{Vv}$ & $1,2,3$ \\
\hline 131 & Psychotria chiapensis Standl. & $\mathrm{C}$ & Aa & Vv & $1,2,3$ \\
\hline 132 & Psychotria costivenia Griseb & $\mathrm{RE}$ & $\mathrm{Ab}$ & Vv & $1,2,3$ \\
\hline 133 & Psychotria pubescens Sw. & $\mathrm{RE}$ & $\mathrm{Ab}$ & Vv & $1,2,3$ \\
\hline 134 & Rondeletia stenosiphon Hemsl. & $\mathrm{C}$ & $\mathrm{Aa}$ & $\mathrm{Vv}$ & $1,2,3$ \\
\hline 135 & $\begin{array}{l}\text { Simira salvadorensis (Standl.) Steyerm. } \\
\text { Rutaceae }\end{array}$ & $\mathrm{C}$ & APA & Vv & $1,2,3$ \\
\hline
\end{tabular}


Apéndice 1. Continúa

\begin{tabular}{|c|c|c|c|c|c|}
\hline Núm. & $\begin{array}{l}\text { Familia } \\
\text { Especies }\end{array}$ & $F I$ & $G F$ & $M D$ & $F C$ \\
\hline 136 & Citrus aurantiifolia (Christm.) Swingle* & MA & $\mathrm{Cu}$ & $\mathrm{H}$ & 1 \\
\hline 137 & Citrus limetta Risso* & MA & $\mathrm{Cu}$ & $\mathrm{H}$ & 1 \\
\hline 138 & Citrus sinensis (L.) Osbeck* & MA & $\mathrm{Cu}$ & $\mathrm{H}$ & 1 \\
\hline 139 & $\begin{array}{l}\text { Zanthoxylum riedelianum Engl. } \\
\text { Sapindaceae }\end{array}$ & $\mathrm{C}$ & APi & $\mathrm{Vv}$ & $1,2,3$ \\
\hline 140 & Cupania dentata DC. & $\mathrm{C}$ & APB & Vnv-v & $1,2,3$ \\
\hline 141 & Sapindus saponaria L. & MA & $\mathrm{APB}$ & Vv & $1,2,3$ \\
\hline 142 & $\begin{array}{l}\text { Talisia oliviformis (Kunth) Radlk. } \\
\text { Sapotaceae }\end{array}$ & $\mathrm{C}$ & APA & Vnv-v & $1,2,3$ \\
\hline 143 & Manilkara chicle (Pittier) Gilly & $\mathrm{C}$ & APA & Vnv-v & $1,2,3$ \\
\hline 144 & Manilkara zapota (L.) P. Royen & $\mathrm{C}$ & APA & Vnv-v & $1,2,3$ \\
\hline 145 & Pouteria campechiana (Kunth) Baehni & $\mathrm{RE}$ & APA & Vnv & $1,2,3$ \\
\hline 146 & Pouteria reticulata (Engl.) Eymma & $\mathrm{C}$ & APA & Vnv & $1,2,3$ \\
\hline 147 & Pouteria sapota (Jacq.) H. E. Moore et Stearn. & $\mathrm{RE}$ & APA & Vnv & $1,2,3$ \\
\hline 148 & Pouteria sp. 1 & $\mathrm{C}$ & APA & Vnv-v & $1,2,3$ \\
\hline 149 & Pouteria sp. 2 & $\mathrm{RE}$ & APA & Vnv-v & $1,2,3$ \\
\hline 150 & Pouteria sp. 3 & $\mathrm{RE}$ & APA & Vnv-v & $1,2,3$ \\
\hline 151 & Sideroxylon aff. foetidissimum (Pittier) T.D. Penn. & $\mathrm{C}$ & APA & Vnv-v & 1 \\
\hline 152 & $\begin{array}{l}\text { Sideroxylon persimile (Hemsl.) T. D. Penn. } \\
\text { Simaroubaceae }\end{array}$ & $\mathrm{RE}$ & APA & Vnv-v & $1,2,3$ \\
\hline 153 & $\begin{array}{l}\text { Simarouba glauca DC. } \\
\text { Sterculiaceae }\end{array}$ & $\mathrm{C}$ & APA & Vnv-v & $1,2,3$ \\
\hline 154 & $\begin{array}{l}\text { Guazuma ulmifolia Lam. } \\
\text { Theaceae }\end{array}$ & MA & APi & Vnv-v & $1,2,3$ \\
\hline 155 & $\begin{array}{l}\text { Ternstroemia tepezapote Schltdl. et Cham. } \\
\text { Tiliaceae }\end{array}$ & MA & $\mathrm{APi}$ & $\mathrm{Vv}$ & 1 \\
\hline 156 & Berrya cubensis (Griseb.) M. Gómez & $\mathrm{RE}$ & APB & $\mathrm{Vv}$ & 1,2 \\
\hline 157 & Heliocarpus appendiculatus Turcz. & $\mathrm{C}$ & $\mathrm{APi}$ & W & 1,3 \\
\hline 158 & Heliocarpus donnell-smithii Rose & $\mathrm{C}$ & $\mathrm{APi}$ & W & $1,2,3$ \\
\hline 159 & Luehea speciosa Willd. & MA & $\mathrm{APi}$ & $\mathrm{W}$ & 1 \\
\hline 160 & Mortoniodendron guatemalense Standl. et Steyerm. & $\mathrm{C}$ & APB & Vnv-v & $1,2,3$ \\
\hline 161 & $\begin{array}{l}\text { Trichospermum mexicanum (DC.) Baill. } \\
\text { Ulmaceae }\end{array}$ & $\mathrm{C}$ & $\mathrm{APi}$ & $\mathrm{G}$ & 1,2 \\
\hline 162 & Ampelocera hottlei (Standl.) Standl. & $\mathrm{C}$ & APA & $\mathrm{Vv}$ & $1,2,3$ \\
\hline 163 & Aphananthe monoica (Hemsl.) Leroy & $\mathrm{C}$ & APA & Vnv-v & $1,2,3$ \\
\hline 164 & $\begin{array}{l}\text { Trema micrantha (L.) Blume } \\
\text { Urticaceae }\end{array}$ & MA & APi & Vnv-v & 1,3 \\
\hline 165 & $\begin{array}{l}\text { Myriocarpa longipes Liebm. } \\
\text { Verbenaceae }\end{array}$ & $\mathrm{C}$ & $\mathrm{Pa}$ & G & $1,2,3$ \\
\hline 166 & Aegiphila monstrosa Moldenke & $\mathrm{C}$ & APA & Vnv-v & $1,2,3$ \\
\hline 167 & Gmelina arborea Roxb.* & MA & $\mathrm{Cu}$ & G & 1 \\
\hline 168 & Lippia cardiostegia Benth. & $\mathrm{C}$ & $\mathrm{Pa}$ & W & $1,2,3$ \\
\hline 169 & Rehdera penninervia Stand. et Moldenke & $\mathrm{C}$ & $\mathrm{P}$ & W & $1,2,3$ \\
\hline 170 & Tectona grandis L.f.* & MA & $\mathrm{Cu}$ & G & 1 \\
\hline 171 & $\begin{array}{l}\text { Vitex gaumeri Greenm. } \\
\text { Violaceae }\end{array}$ & $\mathrm{C}$ & APA & Vnv-v & $1,2,3$ \\
\hline 172 & Rinorea guatemalensis (S. Watson) Bartlett & $\mathrm{C}$ & $\mathrm{Aa}$ & Expl. & $1,2,3$ \\
\hline
\end{tabular}

